This is an open access chapter licensed under the Creative Commons BY-NC 4.0 International License.

\title{
Annex 1: A template for a report from the process of integrated impact assessment on border control technolo- gies in the European Union and the Schengen Area ${ }^{1}$
}

Nikolaos Ioannidis, ${ }^{*}$ Simone Casiraghi, ${ }^{* *}$ Alessandra CAlvi ${ }^{* * *}$ and Dariusz KlozA ${ }^{* * *}$

*Vrije Universiteit Brussel.E-mail: nikolaos.ioannidis@vub.be.

**Vrije Universiteit Brussel.E-mail: simone.casiraghi@vub.be.

${ }^{* * *}$ Vrije Universiteit Brussel. E-mail: alessandra.calvi@vub.be.

****Vrije Universiteit Brussel. E-mail: dariusz.kloza@vub.be.

\section{Cover page}

\begin{tabular}{l} 
Name of the initiative under assessment \\
Name, contact details and other identifying details of: \\
\hline border control authority deploying the initiative \\
\hline data controller(s) \\
\hline data processor(s), if applicable \\
\hline person(s) in charge of the initiative \\
\hline - assessor(s) \\
\hline data protection officer(s) (DPO), if appointed \\
\hline chief information security officer, if appointed \\
\hline
\end{tabular}


- quality control body supervising the assessment process, if appointed

- data protection authority/ies (DPA)

- research ethics committees at public or private organisations

- national ethics committees or councils

- groups of ad hoc recruited ethics experts

- anyone else involved, as practicable

Version of the assessment report

Level of confidentiality of the assessment report

Public

$\square$ Confidential

$\square$ Specific [explain]

Date and place of compilation of the report

[Any other details, as practicable]

\section{Executive summary}

[Summarise the most significant information concerning the outcomes of each step of the integrated impact assessment process.] 


\section{Phase I: preparation of the assessment process}

\section{Step 1: Screening (threshold analysis)}

\section{Step 1a: Preliminary description of the envisaged initiative}

\begin{tabular}{|c|c|c|}
\hline \multirow{7}{*}{ 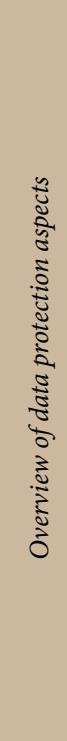 } & \multirow{4}{*}{ 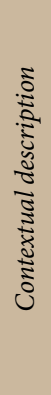 } & What? \\
\hline & & How much/how many? \\
\hline & & Where? \\
\hline & & Why? \\
\hline & \multirow{3}{*}{$\underset{5}{5}$} & $\begin{array}{l}\text { Overview of personal data } \\
\text { and processing operations }\end{array}$ \\
\hline & & Infrastructure \\
\hline & & Actors \\
\hline \multicolumn{3}{|c|}{ Overview of privacy aspects } \\
\hline \multicolumn{3}{|c|}{ Overview of ethical aspects } \\
\hline \multicolumn{3}{|c|}{ Overview of social acceptance aspects } \\
\hline \multicolumn{3}{|c|}{ [other, explain] } \\
\hline
\end{tabular}




\section{Step 1ba: Personal data protection screening (threshold analysis)}

\begin{tabular}{|c|c|c|c|}
\hline Positive criteria & $\begin{array}{c}\text { Legal } \\
\text { provision }\end{array}$ & $\frac{\frac{\pi}{0}}{\frac{\pi}{0}} \frac{\tilde{\pi}}{\frac{\pi}{\pi}}$ & Explanation \\
\hline $\begin{array}{l}\text { Criterion 1: The envisaged processing operations are } \\
\text { likely to result in a high risk to the rights and freedoms of } \\
\text { natural persons (general) }\end{array}$ & $35(1)$ & $\square$ & \\
\hline \multicolumn{4}{|l|}{ Criterion 2: Processing operations deemed highly risky } \\
\hline $\begin{array}{l}\text { 2a. Processing operations entailing systematic and } \\
\text { extensive evaluation of personal aspects relating to } \\
\text { natural persons which is based on automated pro- } \\
\text { cessing, including profiling, and on which decisions } \\
\text { are based that produce legal effects concerning the } \\
\text { natural person or similarly significantly affect the } \\
\text { natural person }\end{array}$ & $35(3)(a)$ & $\square$ & \\
\hline $\begin{array}{l}\text { 2b. Processing operations regarding special categories } \\
\text { of data, or personal data relating to criminal convic- } \\
\text { tions and offences on a large scale }\end{array}$ & $35(3)(b)$ & $\square$ & \\
\hline $\begin{array}{l}\text { 2c. Processing operations entail a systematic monito- } \\
\text { ring of a publicly accessible area on a large scale }\end{array}$ & $35(3)(c)$ & $\square$ & \\
\hline $\begin{array}{l}\text { Criterion 3: Processing operations included in the public } \\
\text { list of processing operations that require a data protection } \\
\text { impact assessment compiled by the DPA(s) to which } \\
\text { jurisdiction(s) the data controller is subject }\end{array}$ & $35(4)$ & $\square$ & \\
\hline $\begin{array}{l}\text { Criterion 3bis: Processing operations that require a } \\
\text { DPIA as included in a code of conduct to which the data } \\
\text { controller is subject }\end{array}$ & 40 & $\square$ & \\
\hline [other, cf. Step 2a: Benchmark; explain] & & $\square$ & \\
\hline \multirow{2}{*}{ DECISION } & & $\square$ & required \\
\hline & & $\square$ & not required \\
\hline
\end{tabular}




\begin{tabular}{|c|c|c|c|}
\hline Negative criteria & $\begin{array}{c}\text { Legal } \\
\text { provision }\end{array}$ & $\frac{\widetilde{a}}{\tilde{3}}$ & Explanation \\
\hline $\begin{array}{l}\text { Criterion 4: Processing operations included in the public } \\
\text { list of processing operations that DO NOT require a data } \\
\text { protection impact assessment compiled by the DPA(s) to } \\
\text { which jurisdiction(s) the data controller is subject }\end{array}$ & $35(5)$ & $\square$ & \\
\hline $\begin{array}{l}\text { Criterion 5: Whereas the legal basis for the processing } \\
\text { operations is the compliance with a legal obligation to } \\
\text { which the controller is subject or the performance of } \\
\text { a task carried out in the public interest, on the basis of } \\
\text { EU or member state's law, and an impact assessment } \\
\text { satisfying the conditions of DPIA under the GDPR has } \\
\text { already been performed }\end{array}$ & $35(10)$ & $\square$ & \\
\hline $\begin{array}{l}\text { Criterion 6: Processing operations concerning personal } \\
\text { data from patients or clients performed by an individual } \\
\text { physician, other health care professional or lawyer }\end{array}$ & Recital 91 & $\square$ & \\
\hline $\begin{array}{l}\text { Criterion 6bis: Processing operations exempted from a } \\
\text { DPIA by a code of conduct to which the data controller } \\
\text { is subject }\end{array}$ & 40 & $\square$ & \\
\hline [other, cf. Step 2a: Benchmark; explain] & & $\square$ & \\
\hline \multirow{2}{*}{ DECISION } & & $\square$ & exempted \\
\hline & & $\square$ & not exempted \\
\hline
\end{tabular}




\section{Step $1 \mathrm{bb}$ : Ethics and social acceptance screening}

Could the initiative result in the development and/or use of technologies and/or processing activities that:

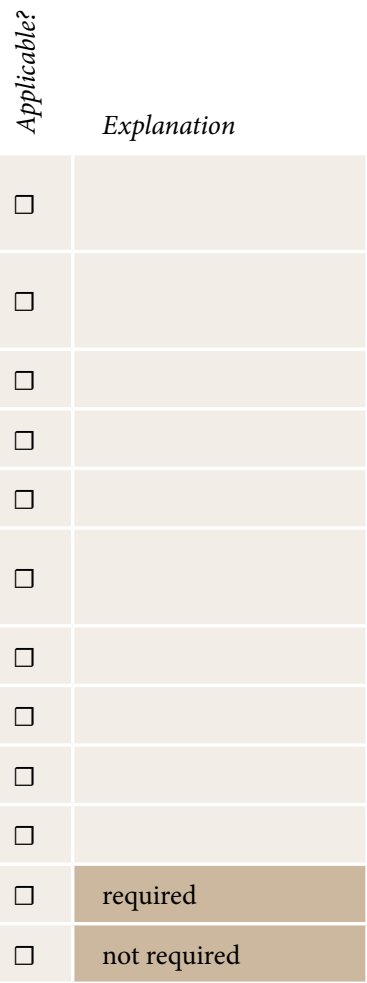

1. Would produce excessive costs in comparison to the advantages they bring?

2. Would fail to ask for the users' consent in a plain understandable language, allowing space for questions, when it is needed?

3. Could be misused (e.g. for terrorism purposes)?

4. Would involve vulnerable individuals or groups?

5. Would involve children and/or minors?

6. Would increase risk of discrimination of certain groups (e.g. third-country nationals)?

7. Would divide users into categories (e.g. low risk and high risk)?

8. Would not be accessible for certain categories of people?

9. Could have potential for military applications?

10. Would increase chances of identity theft? 


\section{Step $1 \mathrm{bc}$ : Privacy screening}

Could the initiative result in the development and/or use of technologies and/or processing activities that:

1. Would interfere with bodily privacy?

2. Would interfere with spatial privacy?

3. Would interfere with communicational privacy?

4. Would interfere with proprietary privacy?

5. Would interfere with intellectual privacy?

6. Would interfere with decisional privacy?

7. Would interfere with associational privacy?

8. Would interfere with behavioural privacy?

9. Would interfere with informational privacy? [overlapping]

RESULT

required

not required

\section{Comments}

[Explanation] 


\section{Step 2: Scoping}

\section{Step 2a: Benchmark}

\section{Step 2aa: Personal data protection}

Applicable laws and regulations

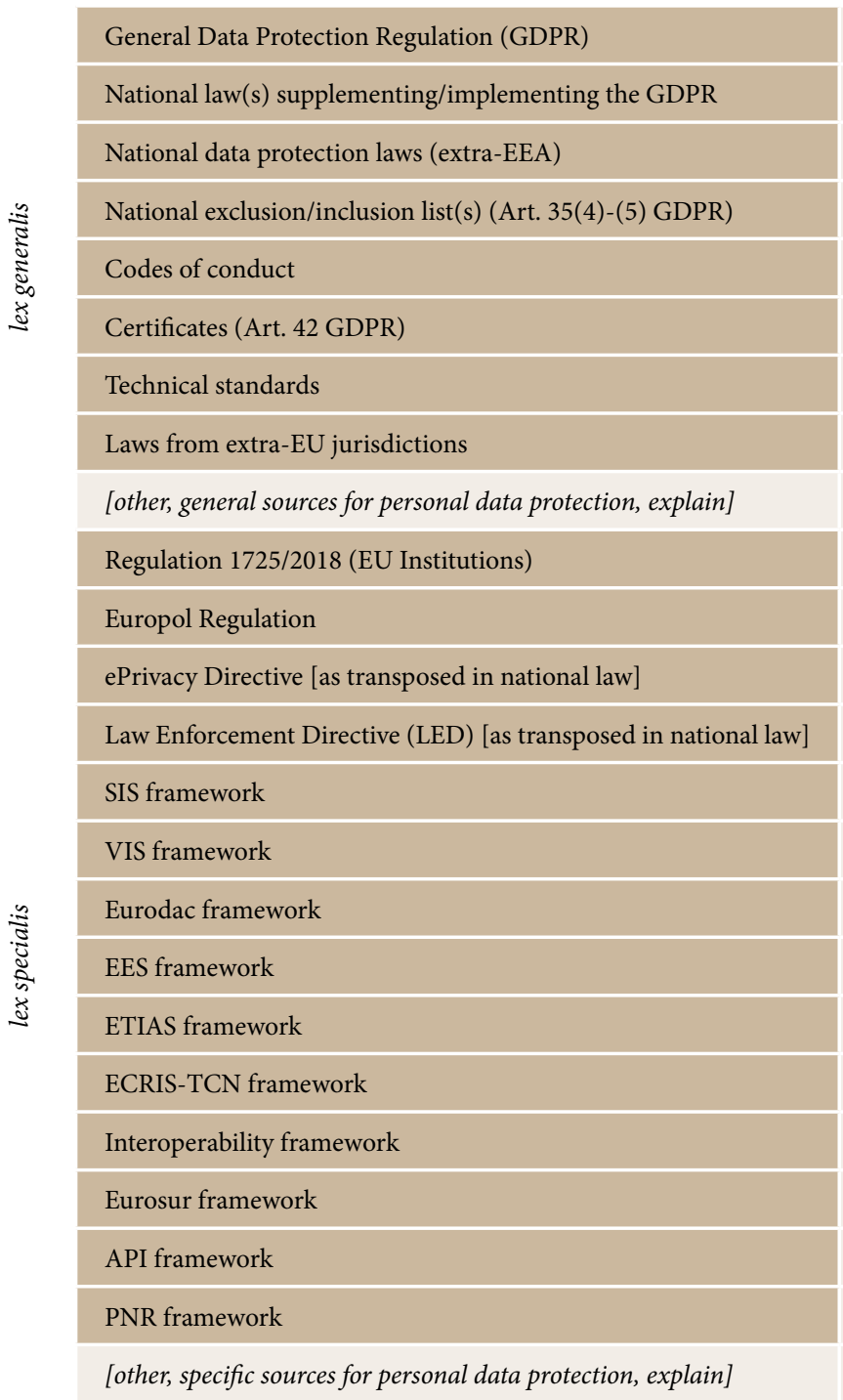


Applicable laws and regulations

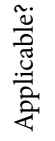

Explanation

$\begin{array}{ll}\frac{\text { है }}{\text { है }} & \text { Data protection policies } \\ \text { डิ } & \text { [other, explain] }\end{array}$

\begin{tabular}{|c|c|c|c|c|}
\hline Scop & f the assessment process & Legal provision & 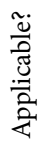 & Explanation \\
\hline \multicolumn{2}{|c|}{ Personal data protection principles } & Art. 5 & $\square$ & \\
\hline \multicolumn{2}{|c|}{ Legal basis for processing } & Art. 6 & $\square$ & \\
\hline \multicolumn{2}{|c|}{ Data subject rights } & Art. $15-22$ & $\square$ & \\
\hline \multicolumn{2}{|c|}{ Obligations of data controller and processor } & Art. 24-39 & $\square$ & \\
\hline \multicolumn{2}{|c|}{ Data transfers outside EU/EEA } & Art. 46 & $\square$ & \\
\hline \multicolumn{2}{|c|}{ Specific processing situations } & Art. $85-91$ & $\square$ & \\
\hline \multirow{6}{*}{ 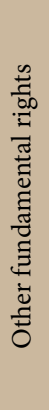 } & $\begin{array}{l}\text { Private and family life, home and } \\
\text { communications }\end{array}$ & \multirow{6}{*}{ Recital 4} & $\square$ & \\
\hline & $\begin{array}{l}\text { Freedom of thought, conscience and } \\
\text { religion }\end{array}$ & & $\square$ & \\
\hline & Freedom of expression and information & & $\square$ & \\
\hline & Freedom to conduct business & & $\square$ & \\
\hline & Right to an effective remedy and to a fair trial & & $\square$ & \\
\hline & Cultural, religious and linguistic diversity & & $\square$ & \\
\hline \multicolumn{3}{|c|}{ [other, explain] } & $\square$ & \\
\hline
\end{tabular}




\section{Step 2ab: Ethics}

\begin{tabular}{|c|c|c|c|c|}
\hline$I D$ & Theory & Argument & Examples & $\frac{8}{4}$ \\
\hline \multirow[t]{3}{*}{1} & \multirow{3}{*}{ ' } & \multirow{3}{*}{$\begin{array}{l}\text { Universality of principles } \\
\text { and/or values }\end{array}$} & 1.1 The initiative is (not) based on universal principles & $\square$ \\
\hline & & & 1.2 The initiative is (not) based on universal values & $\square$ \\
\hline & & & $1 . x \ldots$ & $\square$ \\
\hline \multirow[t]{5}{*}{2} & \multirow{5}{*}{ ' } & \multirow[t]{5}{*}{ Technological determinism } & $\begin{array}{l}2.1 \text { The initiative is presented as a panacea for long-las- } \\
\text { ting social problems }\end{array}$ & $\sqsupset$ \\
\hline & & & $\begin{array}{l}2.2 \text { It is inevitable that the initiative will become ubi- } \\
\text { quitous in society }\end{array}$ & 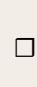 \\
\hline & & & $\begin{array}{l}2.3 \text { It is inevitable that "traditional" border checks will } \\
\text { disappear }\end{array}$ & $\square$ \\
\hline & & & $\begin{array}{l}2.4 \text { The initiative is the only way to solve problems of } \\
\text { security and improve efficiency }\end{array}$ & $\square$ \\
\hline & & & $2 . \mathrm{x} \ldots$ & $\square$ \\
\hline \multirow[t]{3}{*}{3} & \multirow{3}{*}{ ' } & \multirow[t]{3}{*}{ Neutrality of technology } & 3.1 The initiative is (not) neutral & $\square$ \\
\hline & & & 3.2 The initiative is (not) biased & $\square$ \\
\hline & & & $3 . \mathrm{x} \ldots$ & $\square$ \\
\hline \multirow[t]{4}{*}{4} & \multirow{4}{*}{ I } & \multirow[t]{4}{*}{ Arguments from precedent } & $\begin{array}{l}\text { 4.1 The initiative is likely to propose problems that have } \\
\text { happened in the past }\end{array}$ & $\sqsupset$ \\
\hline & & & $\begin{array}{l}4.2 \text { The initiative is likely to solve problems that have } \\
\text { happened in the past }\end{array}$ & $\square$ \\
\hline & & & $\begin{array}{l}\text { 4.3 The initiative is likely to promote benefits that have } \\
\text { happened in the past }\end{array}$ & $\square$ \\
\hline & & & $4 . \mathrm{x} \ldots$ & $\square$ \\
\hline \multirow[t]{5}{*}{5} & \multirow{5}{*}{ ' } & \multirow[t]{5}{*}{$\begin{array}{l}\text { Change of ethical values } \\
\text { arguments }\end{array}$} & $\begin{array}{l}5.1 \text { The initiative will change people's ethical values (such } \\
\text { as autonomy) }\end{array}$ & $\sqsupset$ \\
\hline & & & $\begin{array}{l}5.2 \text { The initiative will change/improve people's ethical } \\
\text { behaviour }\end{array}$ & $\neg$ \\
\hline & & & $\begin{array}{l}5.3 \text { The initiative will change/improve people's ethical } \\
\text { judgements }\end{array}$ & $\square$ \\
\hline & & & $\begin{array}{l}\text { 5.4 The initiative affects the autonomy of border guards' } \\
\text { decision-making }\end{array}$ & $\square$ \\
\hline & & & $5 . x \ldots$ & \\
\hline
\end{tabular}




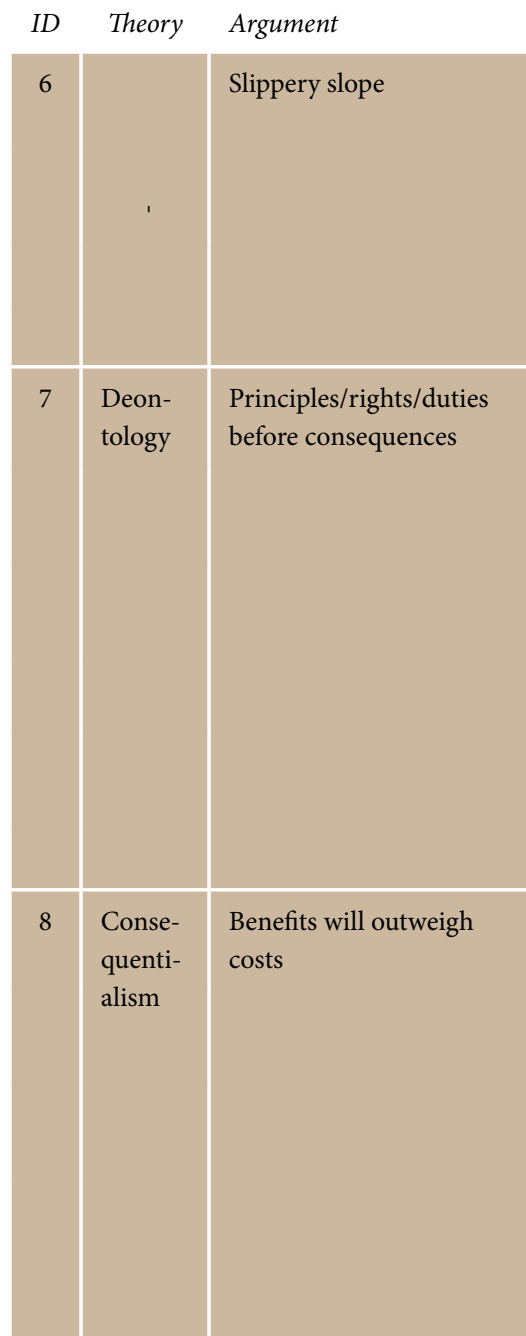

Examples

6.1 The initiative, if developed on a large scale, can give rise to uncontrollable effects

6.2 If we do not implement T now, we will suffer uncontrollable effects

6.3 The initiative bears the risk of "function creep"

6.x $\ldots$

7.1 The initiative will respect principle $\mathrm{X}$, regardless of the consequences

7.2 The initiative is designed respecting the principle/ value $\mathrm{X}$

7.3 There is a categorical prohibition (e.g. "red line”) for certain uses of the initiative

7.4 The initiative (does not) respect the human right $\mathrm{X}$

7.5 The initiative is not in line with the Code of conduct $\mathrm{X}$

7.x $\ldots$

8.1 The initiative brings about (economic) benefits that will outweigh the costs

8.2 The initiative will increase security despite an infringement of privacy

8.3 The initiative will make border crossing/control more efficient

8.4 The initiative can be misused or used for military purposes

8.x ... 


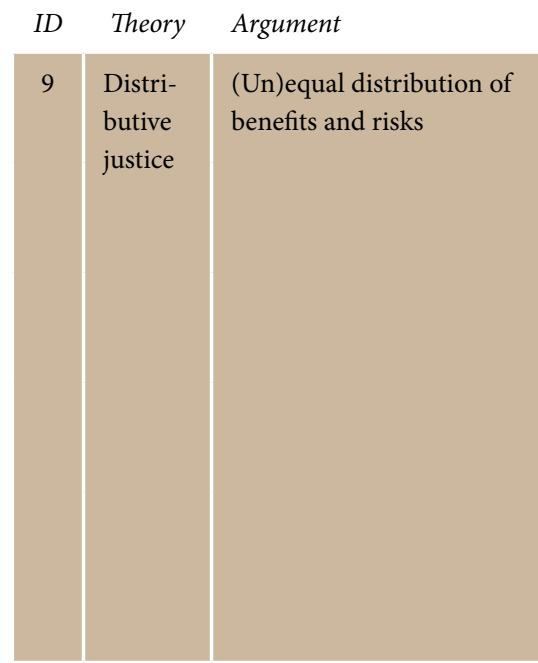

Examples

9.1 The initiative is (not) equally accessible to everyone

(e.g. people in wheelchairs, third-country nationals)

9.2 Only/mostly some people will benefit from the initiative (e.g. bona fide travellers)

9.3 Some people are more prone to be considered highrisk travellers (e.g. third country nationals)

9.4 There are risks of bias or stigmatisation when using the initiative

9.5 The accuracy of the initiative is unreliable for certain categories of people

9.x ...

\section{Step 2ac: Social acceptance scoping}

\begin{tabular}{|c|c|c|c|c|c|}
\hline Perspective & 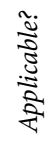 & $\begin{array}{l}\text { Stakeholders considered for } \\
\text { acceptance assessment }\end{array}$ & & $\begin{array}{l}\text { Acceptance assess- } \\
\text { ment technique }\end{array}$ & Explanation \\
\hline \multirow{4}{*}{$\begin{array}{l}\text { Socio- } \\
\text { political }\end{array}$} & \multirow{4}{*}{$\square$} & EU/EEA/CH citizens & $\square$ & & \\
\hline & & $\begin{array}{l}\text { Non-EU/EEA/CH citizens, } \\
\text { and sub-categories }\end{array}$ & $\square$ & & \\
\hline & & Border control authorities & $\square$ & & \\
\hline & & [other, explain] & $\square$ & & \\
\hline \multirow[t]{4}{*}{ Market } & \multirow{4}{*}{$\square$} & Industrial stakeholders & $\square$ & & \\
\hline & & Scientific experts & $\square$ & & \\
\hline & & Policy makers & $\square$ & & \\
\hline & & [other, explain] & $\square$ & & \\
\hline Community & $\square$ & Local stakeholders & $\square$ & & \\
\hline
\end{tabular}


Step 2ad: Privacy

\begin{tabular}{|c|c|c|c|}
\hline \multicolumn{2}{|c|}{ Would it affect...? } & 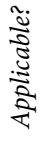 & Explanation \\
\hline \multirow{8}{*}{ 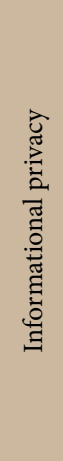 } & Bodily privacy & $\square$ & \\
\hline & Spatial privacy & $\square$ & \\
\hline & Communicational privacy & $\square$ & \\
\hline & Proprietary privacy & $\square$ & \\
\hline & Intellectual privacy & $\square$ & \\
\hline & Decisional privacy & $\square$ & \\
\hline & Associational privacy & $\square$ & \\
\hline & Behavioural privacy & $\square$ & \\
\hline
\end{tabular}

\section{Step 2b: Stakeholders and their consultation techniques}

\section{Internal stakeholders}

\begin{tabular}{|c|c|c|c|c|}
\hline Category of stakeholder & $\begin{array}{l}\tilde{\Xi} \\
\text { בे } \\
\text { دे }\end{array}$ & $\begin{array}{l}\text { Level of } \\
\text { involvement }\end{array}$ & $\begin{array}{l}\text { Stakeholder involve- } \\
\text { ment techniques }\end{array}$ & Explanation \\
\hline Data processor(s) & $\square$ & & & \\
\hline $\begin{array}{l}\text { Data protection officer(s) } \\
\text { (DPO) }\end{array}$ & $\square$ & & & \\
\hline Recipient(s) (Article 4(9)) & $\square$ & & & \\
\hline Third parties (Article $4(10)$ ) & $\square$ & & & \\
\hline Representative(s) (Article 27) & $\square$ & & & \\
\hline Information security officer(s) & $\square$ & & & \\
\hline Legal service & $\square$ & & & \\
\hline $\begin{array}{l}\text { Employees, trade unions, } \\
\text { contractors, etc. }\end{array}$ & $\square$ & & & \\
\hline [other, specify] & & & & \\
\hline
\end{tabular}


Border Control and New Technologies

\section{External stakeholders}

\begin{tabular}{|c|c|c|c|c|c|}
\hline & Category of stakeholder & 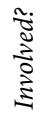 & $\begin{array}{l}\text { Level of } \\
\text { involvement }\end{array}$ & $\begin{array}{l}\text { Stakeholder } \\
\text { involvement } \\
\text { techniques }\end{array}$ & Explanation \\
\hline \multirow{4}{*}{ 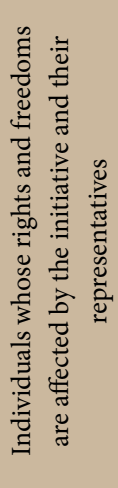 } & $\begin{array}{l}\text { Data subjects, including: } \\
\text { Minors } \\
\text { Vulnerable people } \\
\text { [other, specify] }\end{array}$ & $\square$ & & & \\
\hline & $\begin{array}{l}\text { Representative(s) of data } \\
\text { subject(s) }\end{array}$ & $\square$ & & & \\
\hline & $\begin{array}{l}\text { Individuals who are not data } \\
\text { subjects }\end{array}$ & $\square$ & & & \\
\hline & $\begin{array}{l}\text { Representative(s) of indi- } \\
\text { viduals who are not data } \\
\text { subjects }\end{array}$ & $\square$ & & & \\
\hline \multirow{3}{*}{ 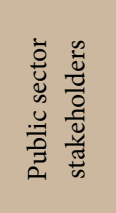 } & $\begin{array}{l}\text { Supervisory authority(ies) } \\
\text { (DPA) }\end{array}$ & $\square$ & & & \\
\hline & Policy makers & $\square$ & & & \\
\hline & Local stakeholders & $\square$ & & & \\
\hline \multirow{2}{*}{ 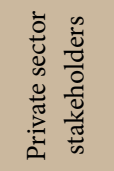 } & Technology providers & $\square$ & & & \\
\hline & Transportation companies & $\square$ & & & \\
\hline \multirow{5}{*}{ 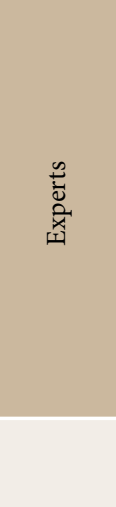 } & $\begin{array}{l}\text { Research Ethics Commit- } \\
\text { tees, at public or private } \\
\text { organisations }\end{array}$ & $\square$ & & & \\
\hline & $\begin{array}{l}\text { National ethics committees } \\
\text { or councils, at EU or Mem- } \\
\text { ber State level }\end{array}$ & $\square$ & & & \\
\hline & $\begin{array}{l}\text { Groups of ad hoc recruited } \\
\text { ethics experts }\end{array}$ & $\square$ & & & \\
\hline & Scientific experts & $\square$ & & & \\
\hline & $\begin{array}{l}\text { [Anybody else affected, etc., } \\
\text { specify] }\end{array}$ & & & & \\
\hline
\end{tabular}




\section{Step 2c: Appraisal techniques}

\begin{tabular}{|c|c|c|c|}
\hline $\begin{array}{l}\text { Element } \\
\text { of the } \\
\text { benchmark }\end{array}$ & Technique & $\frac{\frac{\pi}{3}}{\frac{\pi}{0}}$ & Explanation \\
\hline \multirow{2}{*}{$\begin{array}{c}5 \\
0 \\
0 \\
0 \\
0 \\
0 \\
2 \\
0 \\
0 \\
0 \\
0\end{array}$} & $\begin{array}{l}\text { Necessity and proportionality } \\
\text { assessment }\end{array}$ & $\square$ & \\
\hline & Risk assessment & $\square$ & \\
\hline \multirow{2}{*}{$\begin{array}{l}\grave{\Xi} \\
\text { इे } \\
\text { इ }\end{array}$} & $\begin{array}{l}\text { Necessity and proportionality assess- } \\
\text { ment (as per human rights) }\end{array}$ & $\square$ & \\
\hline & Risk assessment & $\square$ & \\
\hline 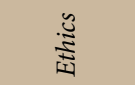 & Ethics assessment & $\square$ & \\
\hline 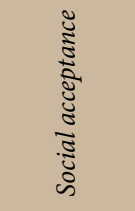 & Social acceptance assessment & $\square$ & \\
\hline 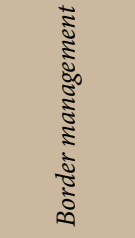 & $\begin{array}{l}\text { Legal compliance with border ma- } \\
\text { nagement law }\end{array}$ & $\square$ & \\
\hline \multirow{4}{*}{ 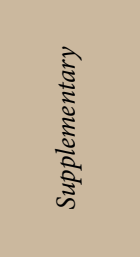 } & Scenario planning & $\square$ & \\
\hline & Cost-Benefit Analysis (CBA) & $\square$ & \\
\hline & $\begin{array}{l}\text { Strengths, Weaknesses, Opportunities, } \\
\text { Threats (SWOT) }\end{array}$ & $\square$ & \\
\hline & [other, specify] & $\square$ & \\
\hline
\end{tabular}


Border Control and New Technologies

Step 2d: Other evaluation techniques

\begin{tabular}{|c|c|c|}
\hline Technique & 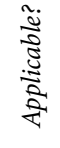 & Explanation \\
\hline Environmental impact assessment & $\square$ & \\
\hline Health impact assessment & $\square$ & \\
\hline Risk assessment & $\square$ & \\
\hline [other, specify] & $\square$ & \\
\hline
\end{tabular}

\section{Comments}

[Explanation] 


\section{Step 3: Planning and Preparation}

\section{Specific objectives of the assessment process}

\begin{tabular}{|c|c|c|}
\hline Objective & 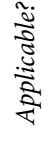 & Explanation \\
\hline Protection of individuals & $\square$ & \\
\hline Compliance with the law & $\square$ & \\
\hline [other, specify] & $\square$ & \\
\hline
\end{tabular}

Criteria for the acceptability of negative impacts

\begin{tabular}{|c|c|c|c|}
\hline \multicolumn{2}{|l|}{ Objective } & 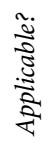 & Explanation \\
\hline \multicolumn{2}{|c|}{ Necessity and proportionality (Article 35(7)(b)) } & $\square$ & \\
\hline \multicolumn{2}{|c|}{$\begin{array}{l}\text { Human rights limitation criteria (Article 52(1) } \\
\text { CFR) }\end{array}$} & $\square$ & \\
\hline \multirow{3}{*}{$\begin{array}{l}\text { Risk assessment } \\
\text { (qualitative, } \\
\text { quantitative) } \\
\text { (risk criteria) }\end{array}$} & Likelihood scale & $\square$ & \\
\hline & Severity scale & $\square$ & \\
\hline & Point of acceptability & $\square$ & \\
\hline \multicolumn{2}{|l|}{ [other, specify] } & $\square$ & \\
\hline
\end{tabular}

\section{Resources}

Value(s)

Explanation

\begin{tabular}{|l|}
\hline $\begin{array}{l}\text { Time } \\
\text { (how long?) }\end{array}$ \\
\hline $\begin{array}{l}\text { Money } \\
\text { (how much?) }\end{array}$ \\
\hline $\begin{array}{l}\text { Workforce } \\
\text { (how many people?) }\end{array}$ \\
\hline $\begin{array}{l}\text { Knowledge } \\
\text { (what expertise?) }\end{array}$ \\
\hline $\begin{array}{l}\text { Know-how } \\
\text { (what experience?) }\end{array}$ \\
\hline
\end{tabular}


Value(s)

Explanation

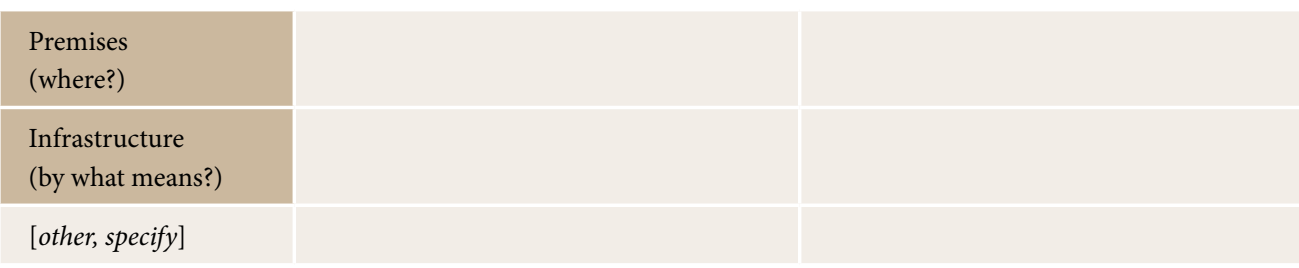

Procedures and timeframes for the assessment process
Milestone
Deadline
Responsibility
Supervision

$1 \quad$ Specify]

2

\section{Assessor(s)}

\begin{tabular}{|c|c|c|c|c|c|c|}
\hline & Name & $\begin{array}{l}\text { If external: } \\
\text { organisation }\end{array}$ & $\begin{array}{l}\text { Contact } \\
\text { details }\end{array}$ & Expertise & $\begin{array}{l}\text { Roles and } \\
\text { responsibilities }\end{array}$ & $\begin{array}{l}\text { Other } \\
\text { information }\end{array}$ \\
\hline 1 & [Specify] & & & & [Leader] & \\
\hline 2 & & & & & & \\
\hline
\end{tabular}

\section{Stakeholders}

[Provide contact details of all stakeholders to involve in the present impact assessment process and a consultation plan, if necessary.]

\section{Continuity of the assessment process}

[How would the present assessment process be continued in the event of a disruption, reorganisation, etc. of the sponsoring organization?] 
Criteria triggering the revision of the assessment process

\begin{tabular}{|c|c|c|}
\hline Criterion & 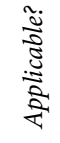 & Explanation \\
\hline Change of likelihood and/or severity of a risk & $\square$ & \\
\hline [Other, specify] & $\square$ & \\
\hline
\end{tabular}

\section{Comments}

[Explanation] 


\section{Ongoing Steps for Phase I}

\section{Step A: Stakeholder involvement}

\section{Internal stakeholders}

\begin{tabular}{|c|c|c|c|}
\hline Category of stakeholders & $\begin{array}{l}\text { What information has } \\
\text { been communicated to } \\
\text { stakeholders? }\end{array}$ & $\begin{array}{l}\text { What input have the } \\
\text { stakeholders provided } \\
\text { (e.g. opinion)? }\end{array}$ & $\begin{array}{l}\text { How was their input } \\
\text { included? Why was it } \\
\text { rejected? }\end{array}$ \\
\hline \multicolumn{4}{|l|}{ Data processor(s) } \\
\hline \multicolumn{4}{|l|}{$\begin{array}{l}\text { Data protection officer(s) } \\
\text { (DPO) }\end{array}$} \\
\hline \multicolumn{4}{|l|}{ Recipient(s) (Article 4(9)) } \\
\hline \multicolumn{4}{|l|}{ Third parties (Article 4(10)) } \\
\hline \multicolumn{4}{|l|}{$\begin{array}{l}\text { Representative(s) } \\
\text { (Article 27) }\end{array}$} \\
\hline \multicolumn{4}{|l|}{$\begin{array}{l}\text { Information security } \\
\text { officer(s) }\end{array}$} \\
\hline \multicolumn{4}{|l|}{ Legal service } \\
\hline \multicolumn{4}{|l|}{$\begin{array}{l}\text { Employees, trade unions, } \\
\text { contractors, etc. }\end{array}$} \\
\hline [other, specify] & & & \\
\hline
\end{tabular}

\section{External stakeholders}

\begin{tabular}{|c|c|c|c|c|}
\hline & Category of stakeholders & $\begin{array}{l}\text { What information } \\
\text { has been communi- } \\
\text { cated to stakeholders? }\end{array}$ & $\begin{array}{l}\text { What input have the } \\
\text { stakeholders provi- } \\
\text { ded (e.g. opinion)? }\end{array}$ & $\begin{array}{l}\text { How was their input } \\
\text { included? Why was } \\
\text { it rejected? }\end{array}$ \\
\hline \multirow{4}{*}{ 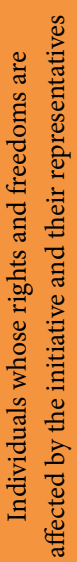 } & $\begin{array}{l}\text { Data subjects, including: } \\
\text { - Minors } \\
\text { Vulnerable people } \\
\text { - }[\text { other, specify] }\end{array}$ & & & \\
\hline & 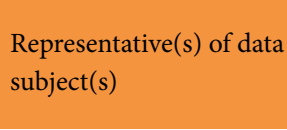 & & & \\
\hline & $\begin{array}{l}\text { Individuals who are not } \\
\text { data subjects }\end{array}$ & & & \\
\hline & $\begin{array}{l}\text { Representative(s) of } \\
\text { individuals who are not } \\
\text { data subjects }\end{array}$ & & & \\
\hline
\end{tabular}




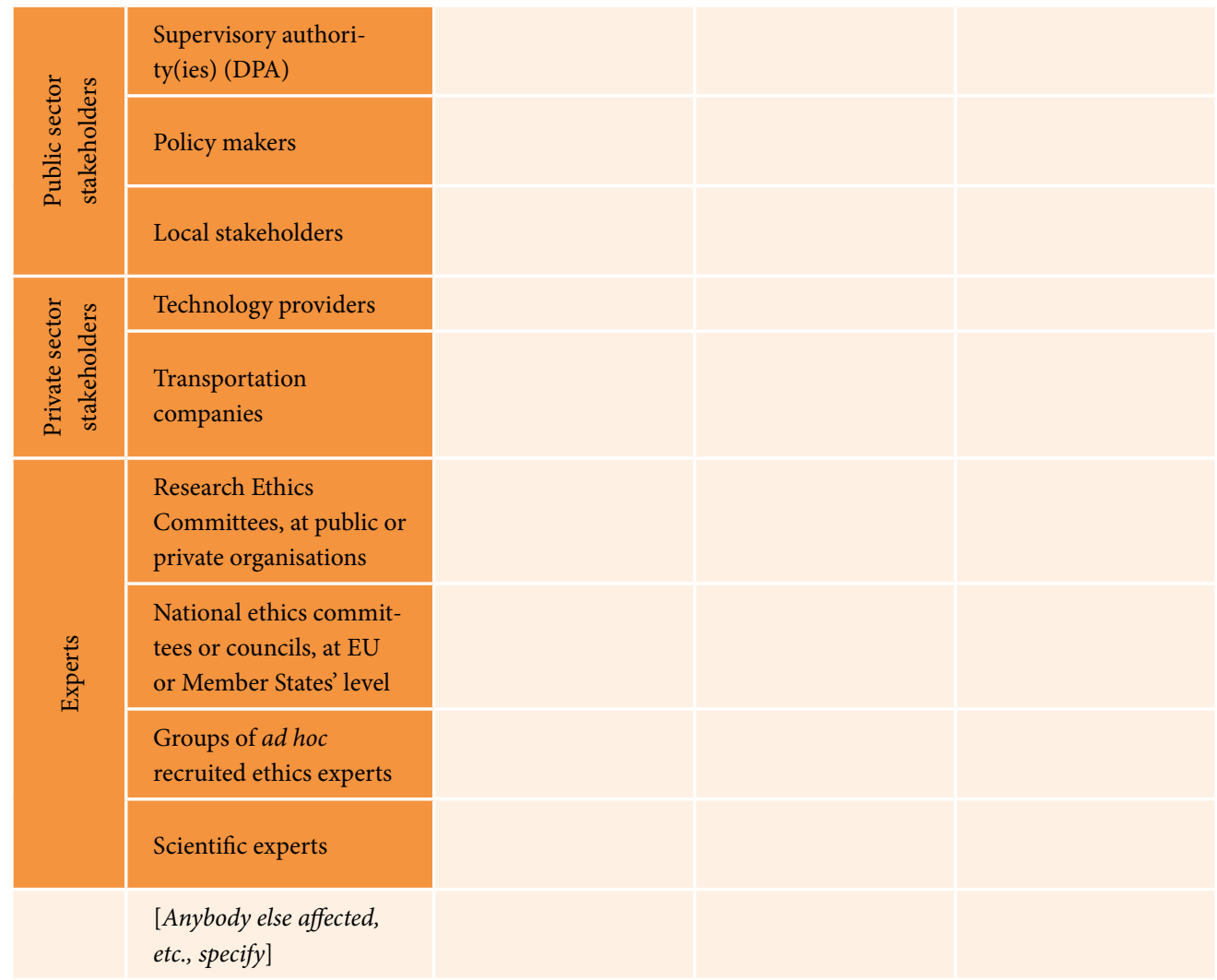

Lack of stakeholder involvement in the present phase

[If stakeholders are not involved in the present phase of the impact assessment process, explain why.] 
Border Control and New Technologies

Step B: Quality control

Quality control body

What feedback was

received?
How was the feedback implemented?

Why was it rejected?

Data protection officer(s) (DPO)

Supervisory authority (DPA)

[Other, specify]

\section{Comments}

[Explanation] 


\section{Phase II: Assessment}

\section{Step 4: Systematic (detailed) description of the initiative}

\section{a) A succinct description of the envisaged initiative}

\section{[Explanation]}

\section{b) Personal data protection}

\section{Overview}

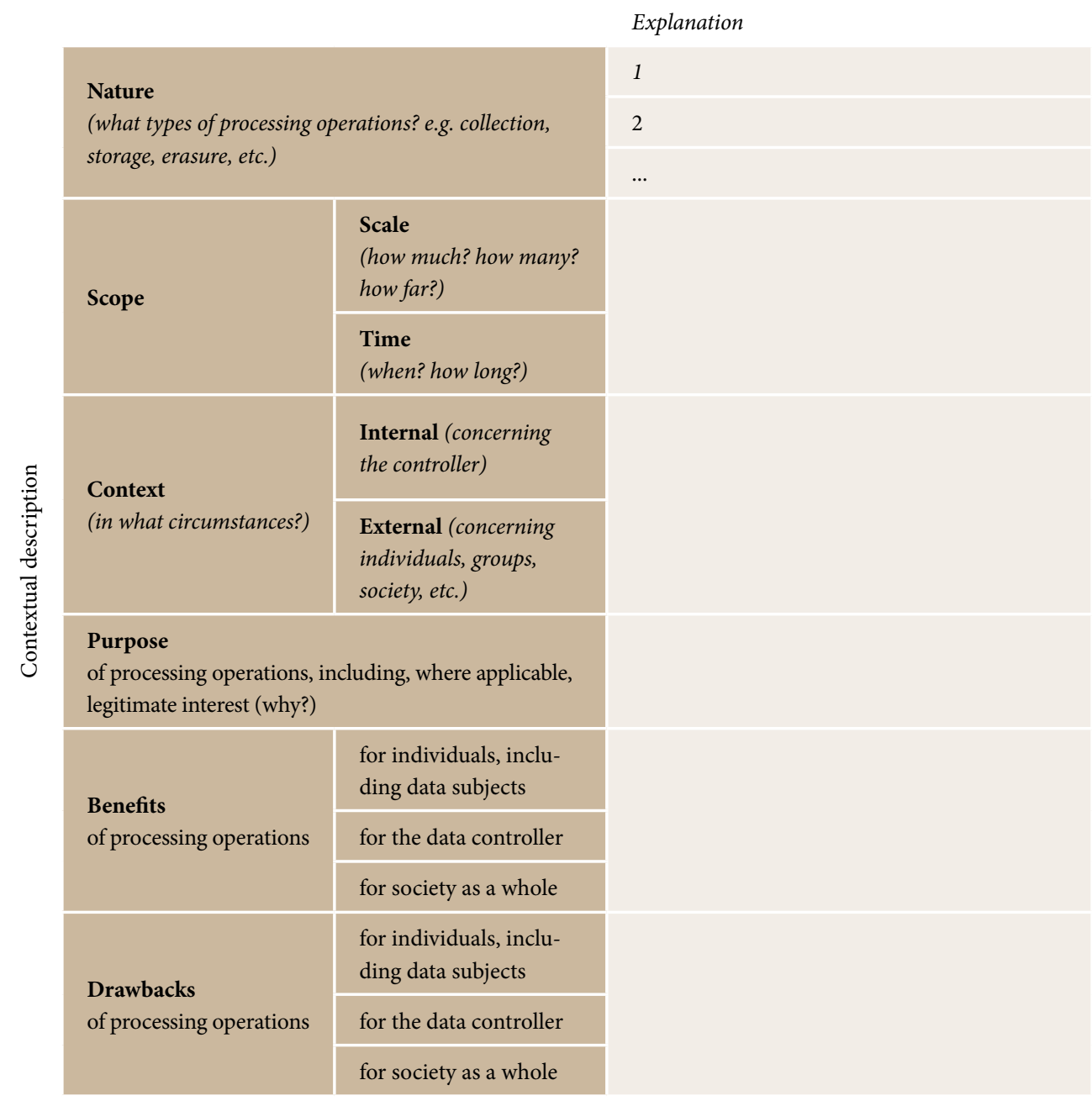




\section{Explanation}

\begin{tabular}{l}
$\begin{array}{l}\text { Categories of personal data (what?) } \\
\text { special categories of personal data } \\
\text { personal data of vulnerable people (e.g. children) } \\
\text { data of a highly personal nature }\end{array}$ \\
\begin{tabular}{l} 
Means of processing (infrastructure) (by what means?) \\
\hline whom?)
\end{tabular} \\
\hline Data security (how is it ensured?) \\
\hline Actors in the 'supply chain' (who?) \\
\hline [Other, explain]
\end{tabular}

Diagram of personal data flows and/or other visualisations

[Insert a diagram] 


\section{c) Privacy}

\section{Explanation}

Bodily privacy
Spatial privacy
Communicational privacy
Proprietary privacy
Intellectual privacy
Decisional privacy
Associational privacy
Behavioural privacy
Informational privacy

\section{Comments}

[Explanation] 
Border Control and New Technologies

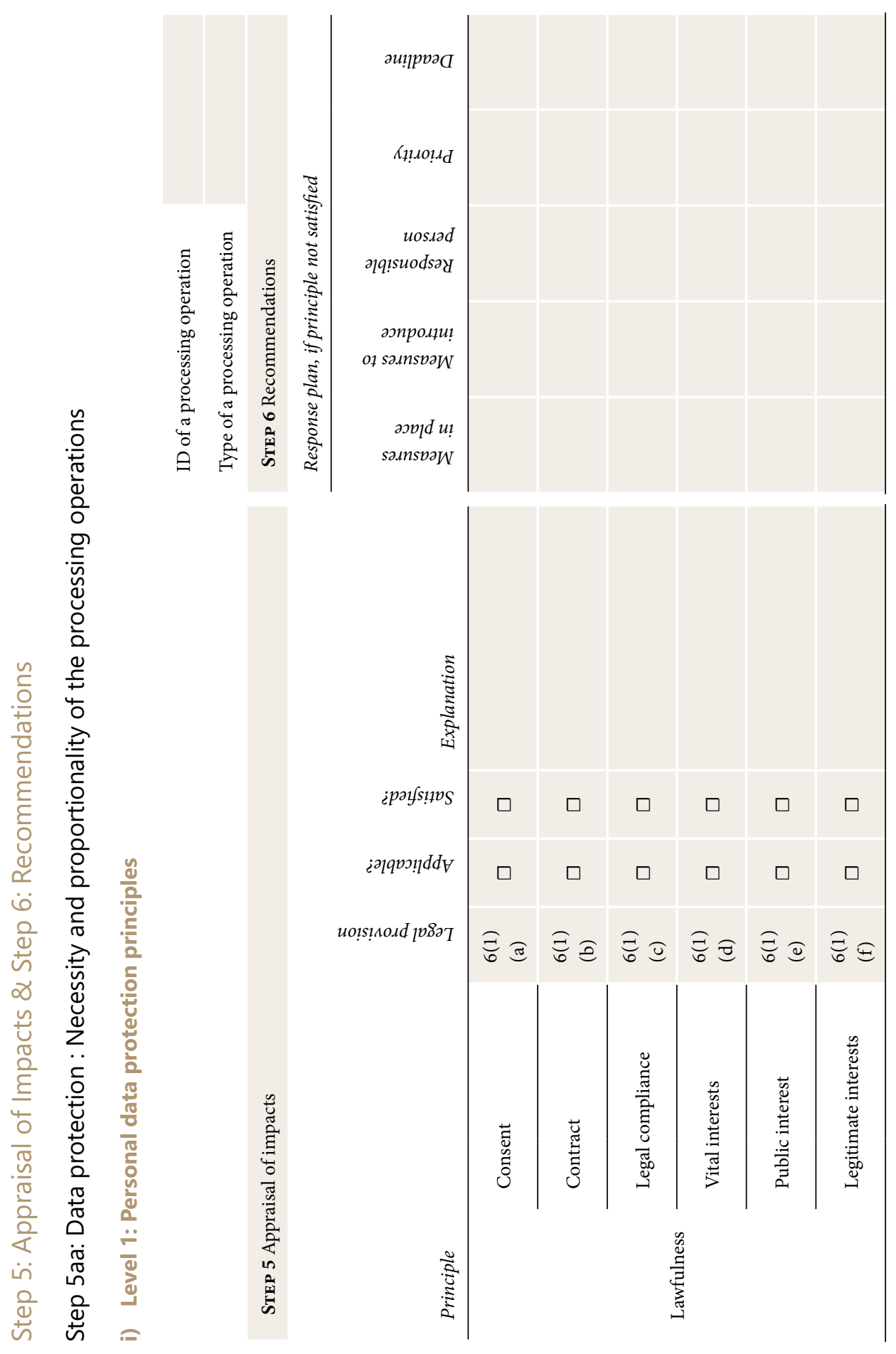


Annex 1 - Step 5: Appraisal of Impacts

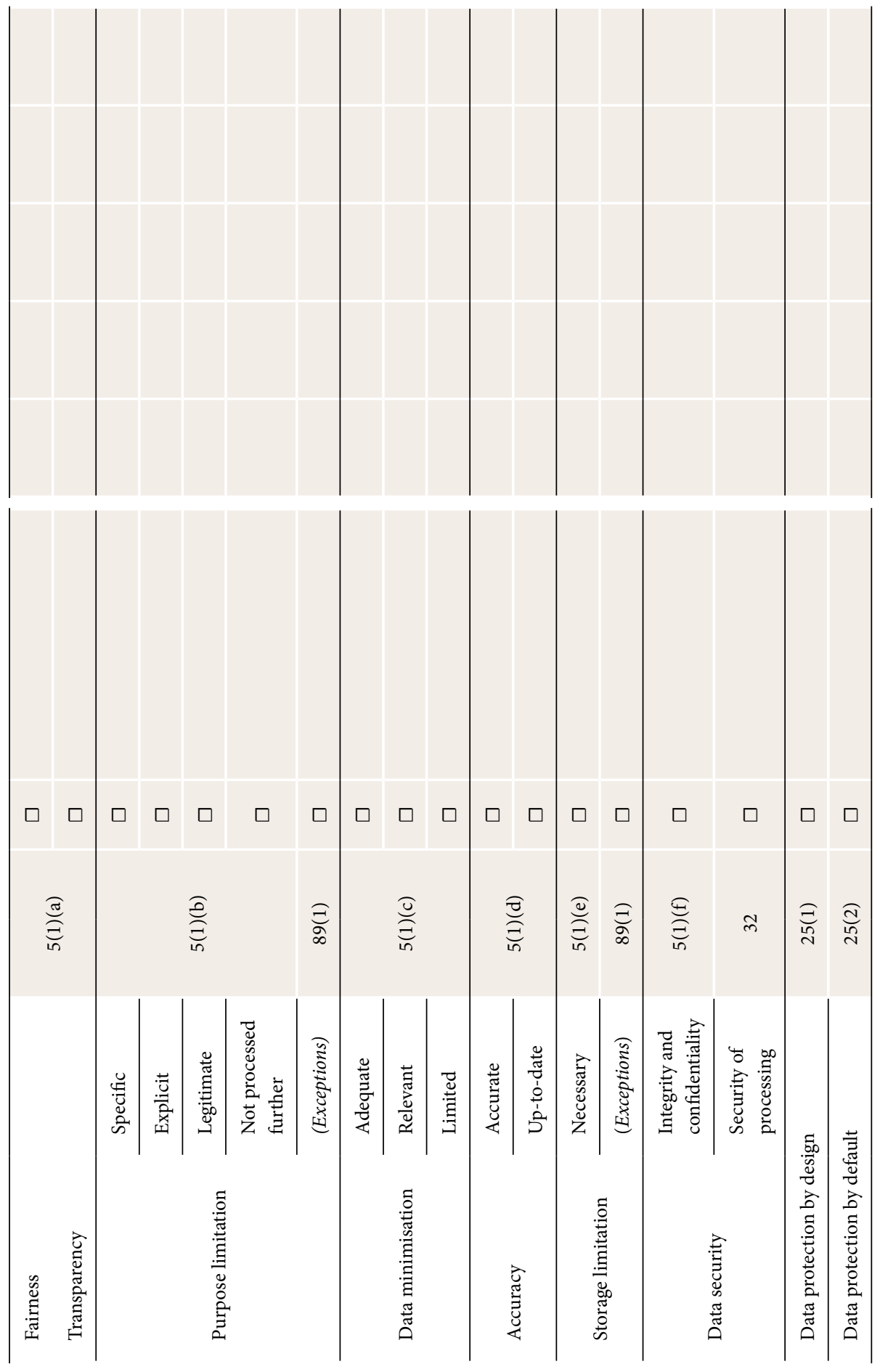


Border Control and New Technologies
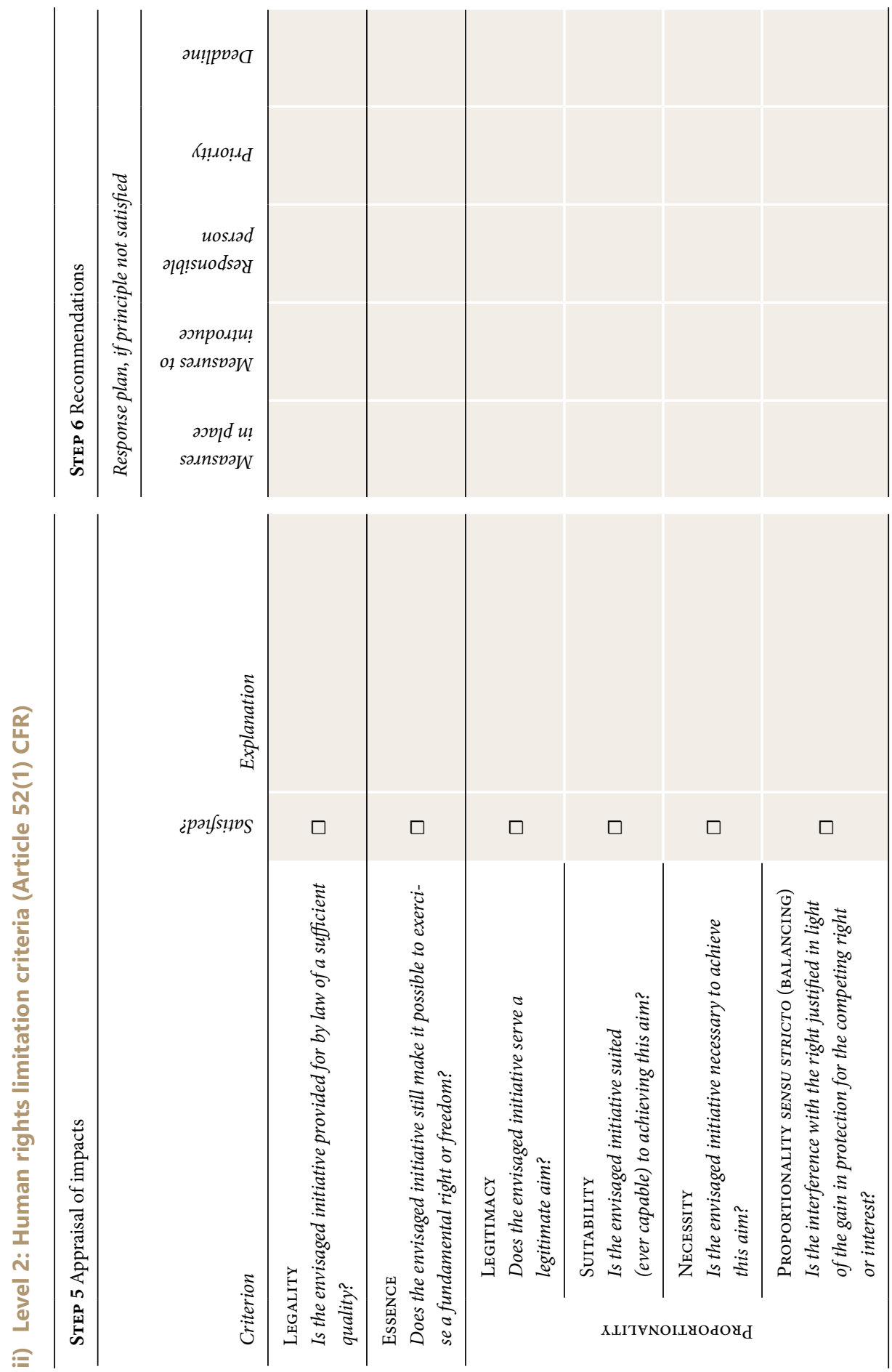
Annex 1 - Step 5: Appraisal of Impacts

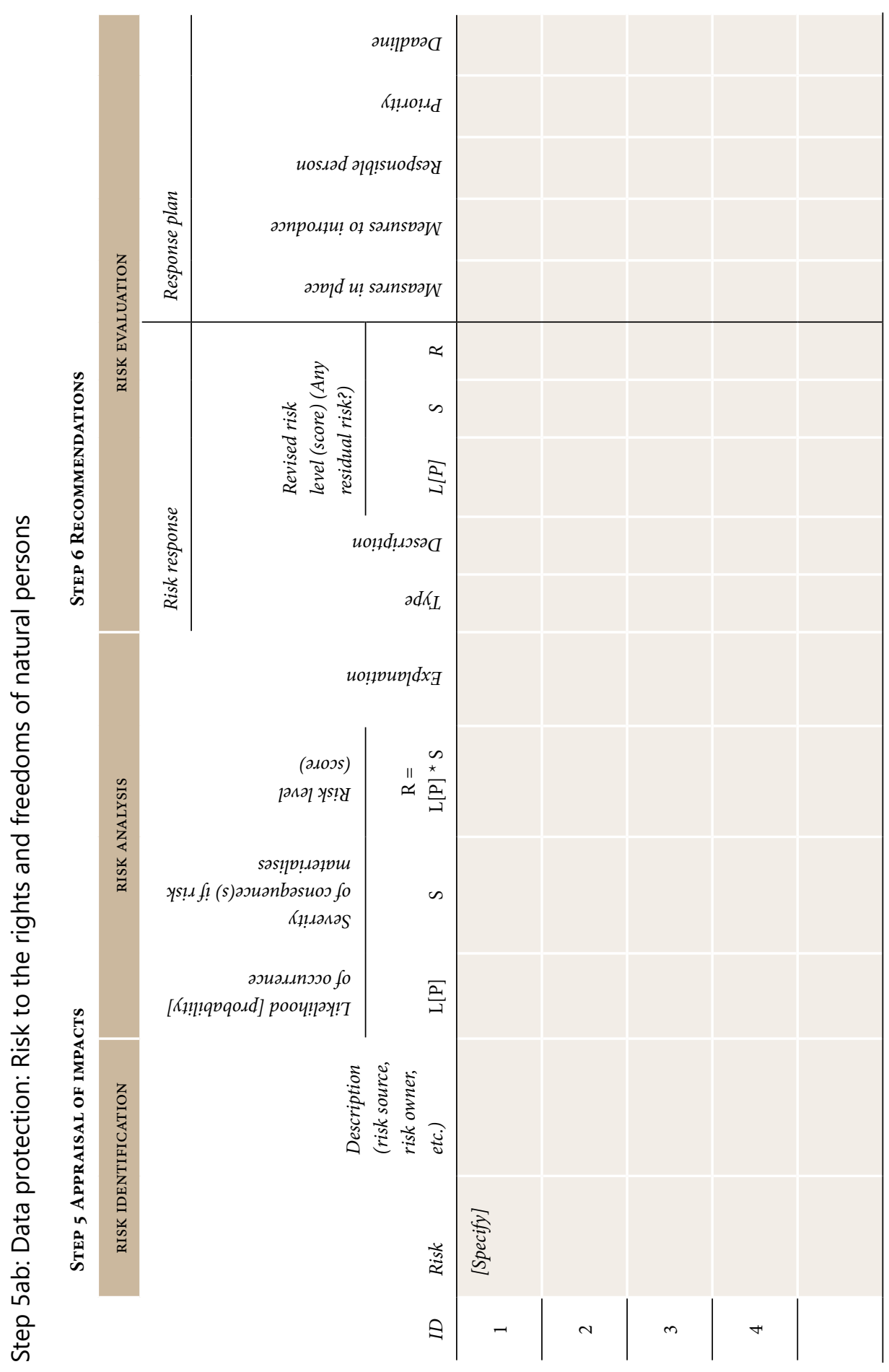


Border Control and New Technologies

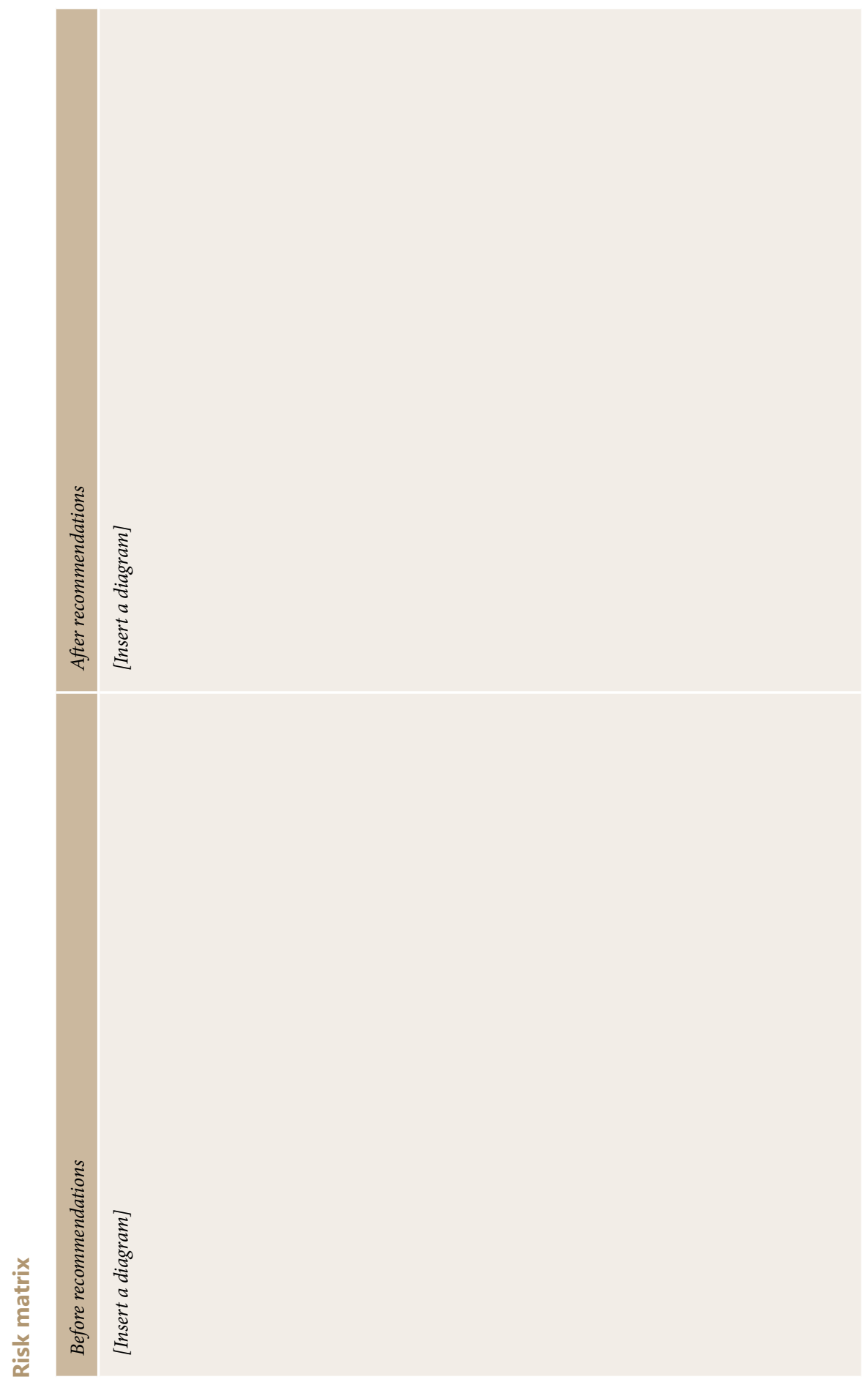




\section{Step 5b: Ethics assessment}

\section{Stage 1: Analysis}

\begin{tabular}{|c|c|c|}
\hline ID & Questions & Answers \\
\hline 1 & How is the initiative (not) in line with universal values or principles? & \\
\hline 2 & $\begin{array}{l}\text { How is the initiative presented in a deterministic way? Is it a positive } \\
\text { or negative picture? }\end{array}$ & \\
\hline 3 & Why is the initiative (not) neutral? & \\
\hline 4 & $\begin{array}{l}\text { Is the initiative legitimised by similar technologies that already } \\
\text { worked in the past? Or is it legitimised by reference to a dystopian } \\
\text { future? }\end{array}$ & \\
\hline 5 & How is the initiative said to change our values or ethical principles? & \\
\hline 6 & $\begin{array}{l}\text { How does the use (or lack of use) of the initiative cause uncontrol- } \\
\text { lable effects? }\end{array}$ & \\
\hline 7 & $\begin{array}{l}\text { How does the initiative protect principles/rights/duties before con- } \\
\text { sequences? Which principles/rights/duties are respected, and which } \\
\text { are infringed? }\end{array}$ & \\
\hline 8 & $\begin{array}{l}\text { Why is the initiative said to produce more benefits than costs? How } \\
\text { is the argument justified? }\end{array}$ & \\
\hline 9 & $\begin{array}{l}\text { How are the risks and benefits of the initiative distributed between } \\
\text { different groups? Which groups are discriminated and how? }\end{array}$ & \\
\hline
\end{tabular}

\section{Stage 2: Assessment}

Assessment

\begin{tabular}{|c|c|c|c|}
\hline IDs & Questions & Conflict & Counterarguments or fallacies \\
\hline 1 & $\begin{array}{l}\text { Are the values/principles } \\
\text { invoked universal? Or are they } \\
\text { instead local? }\end{array}$ & & \\
\hline 2 & $\begin{array}{l}\text { Will the initiative materialise } \\
\text { independently of what people } \\
\text { think and decide? } \\
\text { Or is there some room for } \\
\text { alternatives? }\end{array}$ & & \\
\hline 3 & $\begin{array}{l}\text { Is the initiative neutral or } \\
\text { biased? }\end{array}$ & & \\
\hline 4 & $\begin{array}{l}\text { Does the parallel with the past/ } \\
\text { future hold? }\end{array}$ & & \\
\hline
\end{tabular}


To what extent does the initiative change our morality?

Can more and more similar initiatives ultimately lead to a dystopian future if used on a larger scale, although it seems innocuous at first?

Do the principles/rights/duties invoked actually justify the initiative?

Are invocations to principles/ rights/duties side-tracked by consequentialist arguments? Can one principle/right/duty be outweighed by another? If so, how do you balance competing principles?

Are the promises of the initiative plausible?

Is there a better alternative to the initiative (e.g. less invasive) that is technically and economically feasible?

What are the possible unintended side effects?

Do costs outweigh benefits? Or are the costs and risks downplayed?

Is (the access to) the initiative distributed equally between travellers?

Is (the access to) the initiative distributed on the basis of the needs of the travellers? Are distributive justice arguments side-tracked by consequentialist ones? Are discriminatory issues sufficiently addressed? 


\section{Step 5c: Social acceptance assessment}

\section{Stage 1: Analysis}

\begin{tabular}{|c|c|c|c|c|}
\hline$I D$ & $\begin{array}{l}\text { Acceptance assessment } \\
\text { technique }\end{array}$ & Type of analysis & & Findings and patterns (summary) \\
\hline \multirow[t]{3}{*}{1} & \multirow[t]{3}{*}{$\ldots$} & Quantitative & $\square$ & \\
\hline & & Qualitative & $\square$ & \\
\hline & & Mix & $\square$ & \\
\hline
\end{tabular}

\section{Stage 2: Assessment}

\begin{tabular}{|c|c|c|}
\hline$I D$ & $\begin{array}{l}\text { Positive or negative } \\
\text { consequences }\end{array}$ & Stakeholders affected \\
\hline \multirow[t]{3}{*}{1} & $1 \mathrm{x} \ldots$ & \\
\hline & $1 y \ldots$ & \\
\hline & $1 z \ldots$ & \\
\hline
\end{tabular}

\section{Step 5d: Privacy assessment}

Technology implemented (repeat and justify for each)

\begin{tabular}{|l|}
\hline Bodily privacy \\
\hline Spatial privacy \\
\hline Communicational privacy \\
\hline Proprietary privacy \\
\hline Intellectual privacy \\
\hline Decisional privacy \\
\hline Associational privacy \\
\hline Behavioural privacy \\
\hline
\end{tabular}

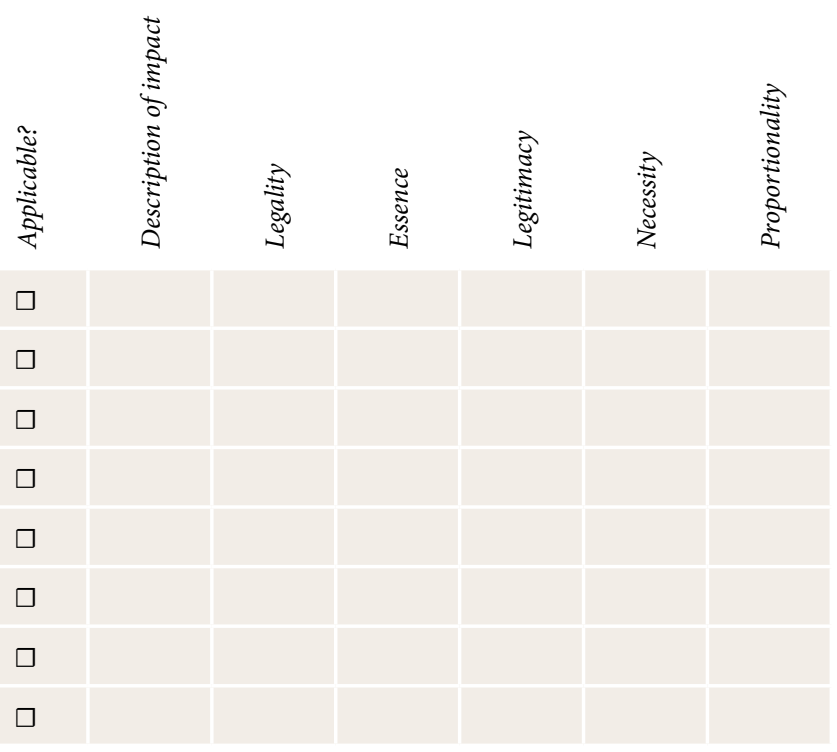


Step 5e: Legal compliance requirements

ID Description

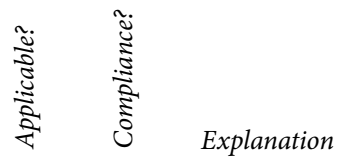

\begin{tabular}{|c|c|c|c|c|}
\hline & \multicolumn{4}{|c|}{ Data Protection } \\
\hline \multirow[t]{2}{*}{1} & \multirow[t]{2}{*}{$\begin{array}{l}\text { Roles of controllers } \\
\text { and processors }\end{array}$} & $\begin{array}{l}\text { Have the responsibilities of } \\
\text { controllers and processors } \\
\text { been allocated in accordance } \\
\text { with the law? }\end{array}$ & $\square$ & $\square$ \\
\hline & & [other, specify] & & \\
\hline \multirow[t]{2}{*}{2} & \multirow[t]{2}{*}{ Lawful processing } & $\begin{array}{l}\text { Has a legal basis grounding } \\
\text { the personal data processing } \\
\text { been identified? }\end{array}$ & $\square$ & $\square$ \\
\hline & & [other, specify] & & \\
\hline \multirow[t]{2}{*}{3} & \multirow[t]{2}{*}{ Purpose limitation } & $\begin{array}{l}\text { Are the purposes for which } \\
\text { a border control technology } \\
\text { processes personal data in } \\
\text { line with those specified in } \\
\text { the relevant legal and other- } \\
\text { wise regulatory framework } \\
\text { applicable to it? }\end{array}$ & $\square$ & $\square$ \\
\hline & & [other, specify] & & \\
\hline \multirow{3}{*}{4} & \multirow{3}{*}{ Data minimisation } & $\begin{array}{l}\text { 1. Does the border control } \\
\text { technology process only } \\
\text { the personal data that is } \\
\text { adequate, relevant and } \\
\text { not excessive for the } \\
\text { specific border control } \\
\text { activity? }\end{array}$ & $\square$ & $\square$ \\
\hline & & $\begin{array}{l}\text { 2. Does the border control } \\
\text { technology ensure that } \\
\text { only specific categories } \\
\text { of personal data are } \\
\text { processed? }\end{array}$ & $\square$ & $\square$ \\
\hline & & [other, specify] & & \\
\hline
\end{tabular}




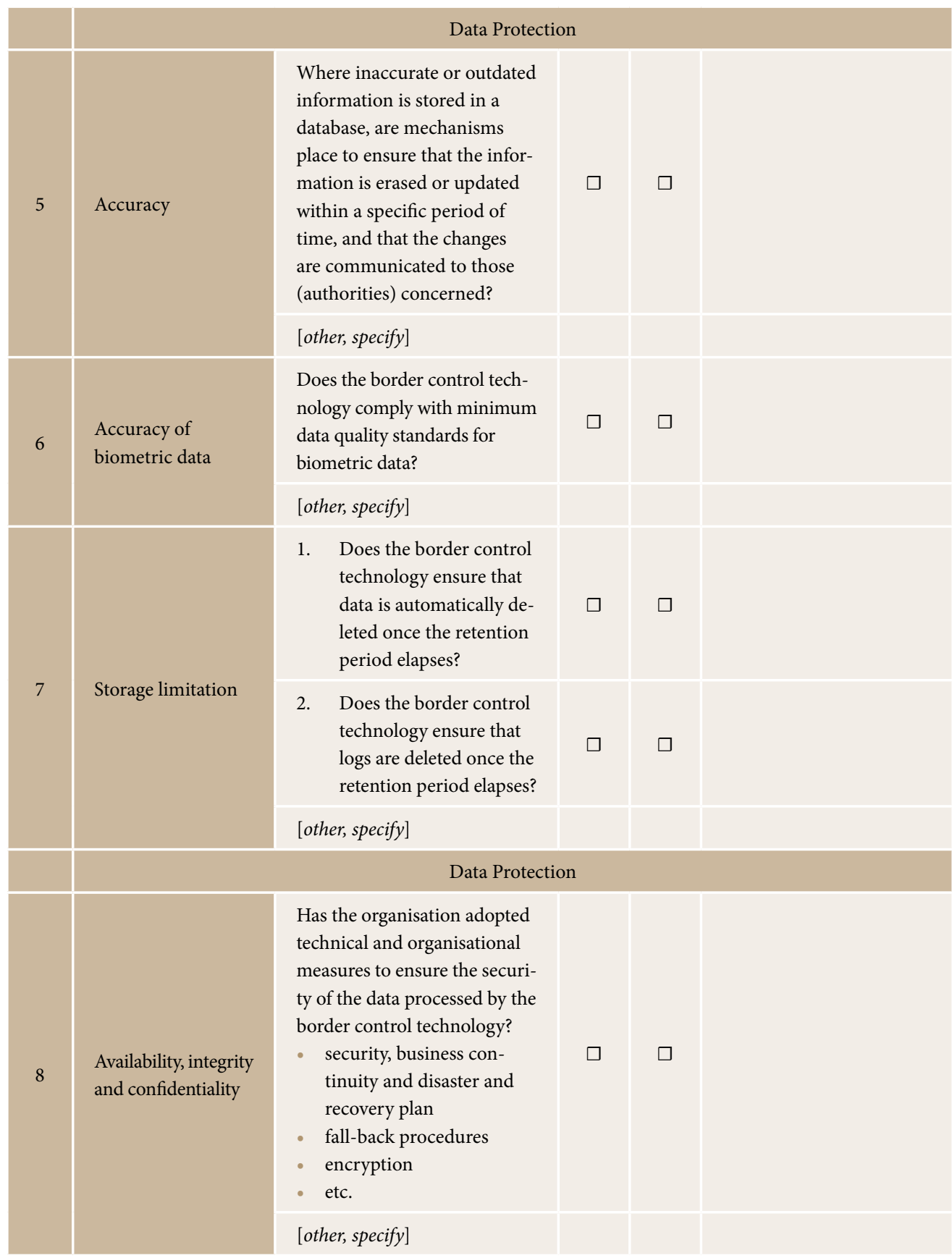




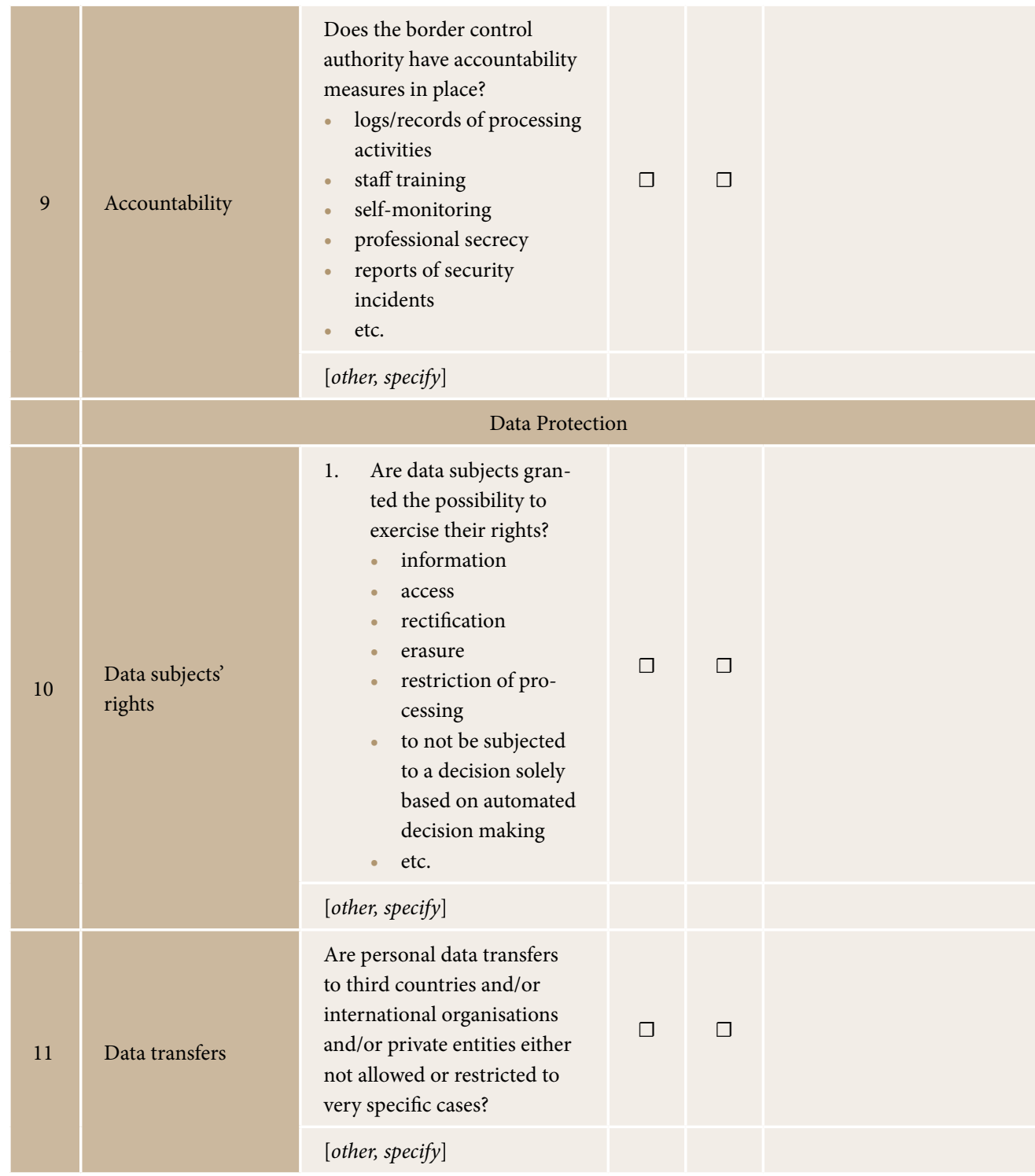




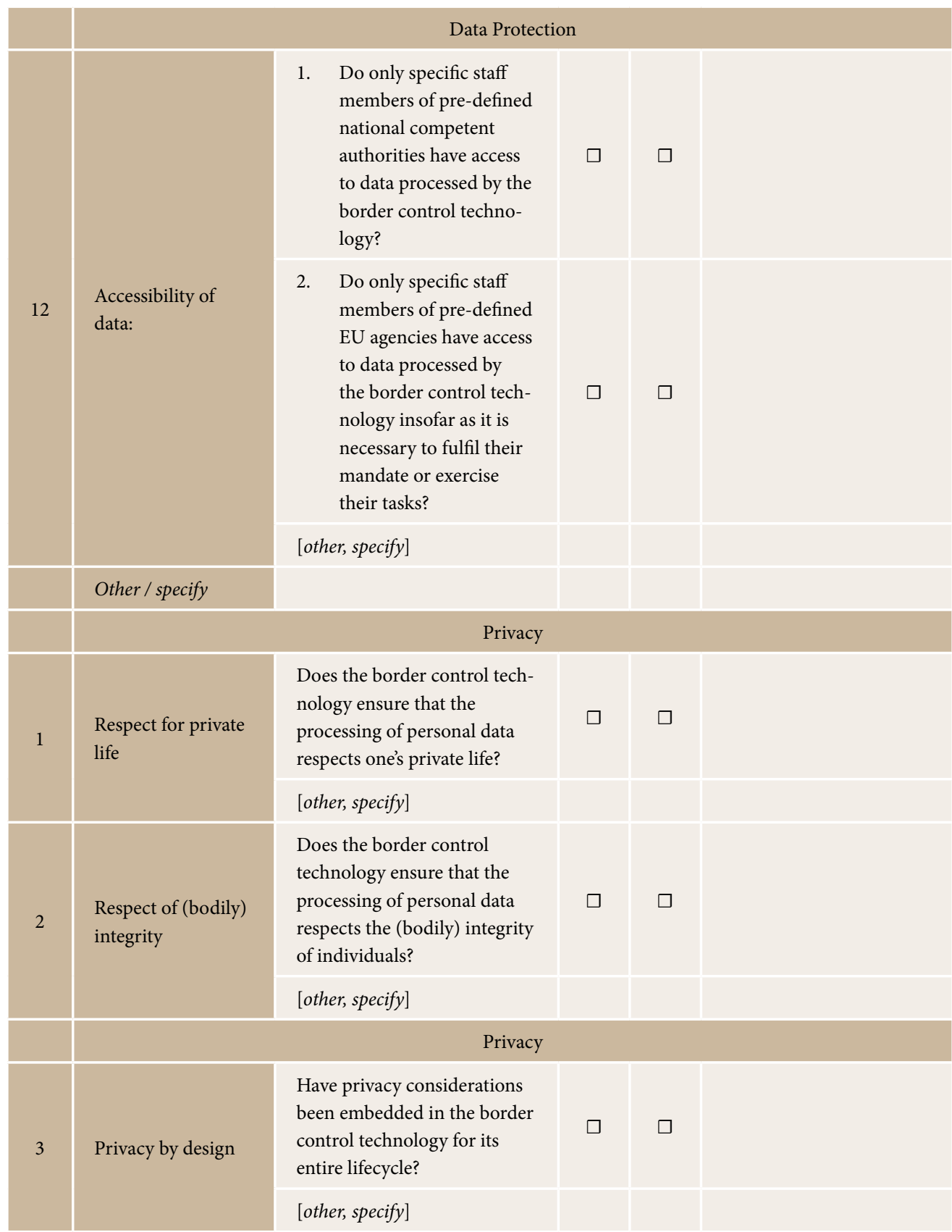




\begin{tabular}{|l|l}
\hline ID & Description \\
\hline 4 & Privacy by default \\
\hline & Other / specify
\end{tabular}

Informed consent

2

Freedom of choice

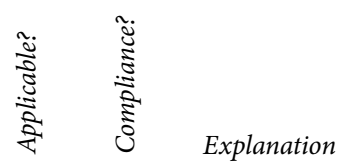

Are the default settings of the border control technology the most privacy-friendly possible?

[other, specify]

\section{Ethics}

1. Is the public informed about the existence of the border crossing point?

2. Is the public informed of the temporary reintroduction of border controls?

[other, specify]

1. May a person opt to not use a border control technology (e.g. e-gate)?

2. Are persons who opt to not use the border control technology not discriminated against for their choice?

[other, specify]

\section{Ethics}

Are restrictions in place for dual-use items?

\section{Other / specify}

Is the use of the border control technology fair towards third-country nationals?

[other, specify] 
ID Description

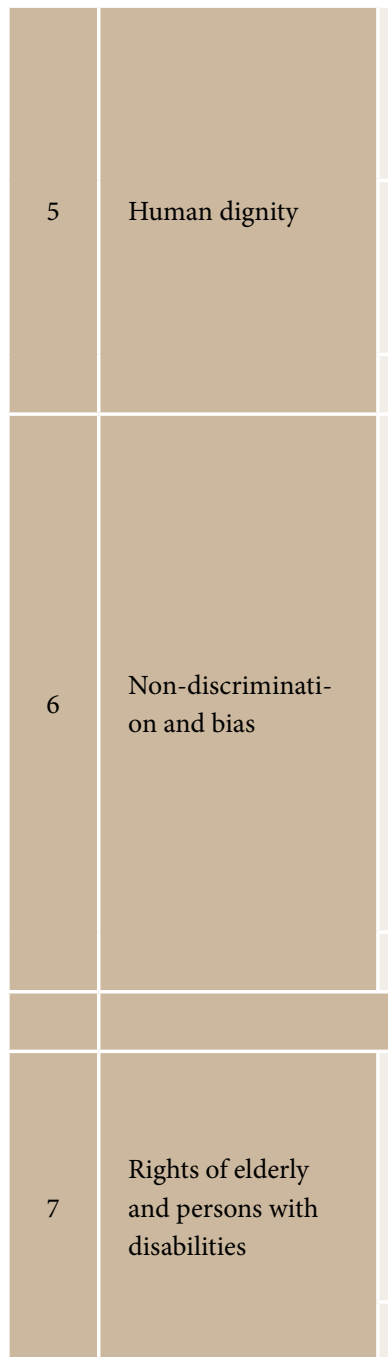

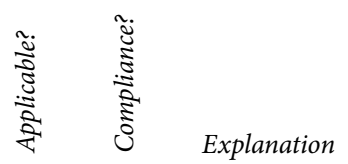

1. Does the use of the border control technology not result in inhuman or degrading treatment?

2. Is the procedure of taking fingerprints in accordance with safeguards in CFR?

[other, specify]

Has the technology been developed in such a way that the processing of personal data will not result in discrimination against persons on any grounds, such as gender, race, colour, ethnic or social origin, genetic features, language, religion or belief, political or any other opinion, membership of a national minority, property, birth, disability, age or sexual orientation?

[other, specify]

\section{Ethics}

Has the border control technology been designed in such a way to be used by all persons, except for children under 12 years of age, to the fullest extent possible?

[other, specify] 


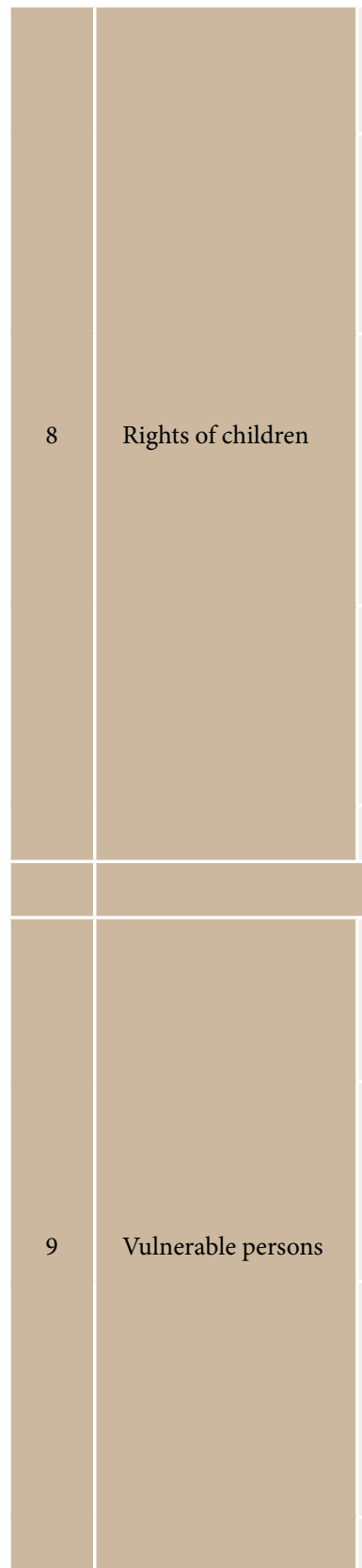

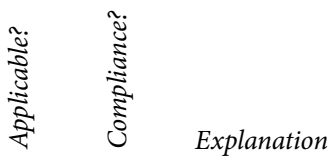

1. Are children under a certain age exempted from giving fingerprints?

2. Are alerts regarding children admissible only in restricted cases and to safeguard the best interest of the child?

3. Are alerts concerning children deleted when the child reaches the age of majority and in the circumstances specified in Article 55 SIS Regulation 1862 ?

4. Are queries in the CIR against minors of 12 years or under allowed, except when in the best interest of the child?

[other, specify]

1. Are alerts concerning vulnerable persons admissible only in restricted cases?

2. Are the alerts concerning vulnerable persons deleted in the circumstances specified in Article 55 SIS Regulation 1862?

3. Have border guards received specialised training for detecting and dealing with situations involving vulnerable persons?

[other, specify] 


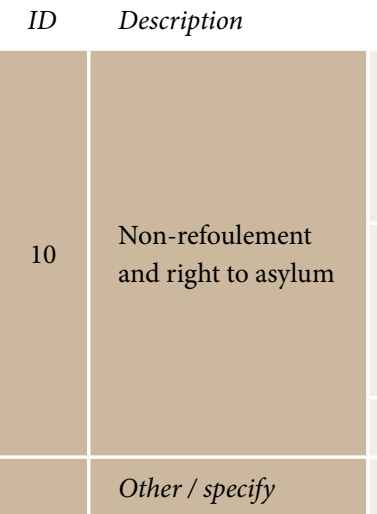

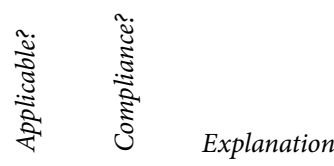

1. Are the individuals not subject to refoulement? Do they have the possibility to ask for asylum?

2. Are the rights of people in need of international protection taken into special account?

[other, specify]

\section{Other evaluation techniques}

Assessment

\begin{tabular}{|l|l|}
\hline [Explanation] & [Explanation] \\
\hline & \\
\hline
\end{tabular}

\section{Comments}

[Explanation] 


\section{Step 6: Recommendations}

Recommendations concerning ethics

\begin{tabular}{|c|c|c|c|c|c|c|}
\hline \multirow[b]{2}{*}{$I D$} & \multirow[b]{2}{*}{ Conflicts } & \multirow{2}{*}{$\begin{array}{l}\text { Counter- } \\
\text { arguments }\end{array}$} & \multirow[b]{2}{*}{ Fallacies } & \multicolumn{3}{|c|}{ Response plan } \\
\hline & & & & Measure & Responsible & Deadline \\
\hline 1 & & & & & & \\
\hline & & & & & & \\
\hline
\end{tabular}

Recommendations concerning social acceptance

\begin{tabular}{|c|c|c|c|c|c|}
\hline \multirow[b]{2}{*}{$I D$} & \multirow[b]{2}{*}{ Users } & \multirow[b]{2}{*}{ Critical points } & \multicolumn{3}{|c|}{ Response plan } \\
\hline & & & Measure & Responsible & Deadline \\
\hline 1 & & & & & \\
\hline & & & & & \\
\hline
\end{tabular}

\section{Recommendations concerning privacy}

\begin{tabular}{|c|c|c|c|c|c|}
\hline \multirow[b]{2}{*}{ Technology } & \multirow{2}{*}{$\begin{array}{l}\text { Aspect(s) of } \\
\text { privacy }\end{array}$} & \multirow[b]{2}{*}{ Interference } & \multicolumn{3}{|c|}{ Response plan } \\
\hline & & & Measure & Responsible & Deadline \\
\hline
\end{tabular}

\section{Recommendations concerning legal compliance}

\begin{tabular}{|c|c|c|c|}
\hline \multicolumn{4}{|c|}{ P } \\
\hline$I D$ & Measure & Responsible & Deadline \\
\hline \multicolumn{4}{|c|}{ Data protection } \\
\hline \multicolumn{4}{|l|}{1} \\
\hline \multicolumn{4}{|c|}{ Privacy } \\
\hline \multicolumn{4}{|l|}{1} \\
\hline \multicolumn{4}{|c|}{ Ethics } \\
\hline 1 & & & \\
\hline & & & \\
\hline
\end{tabular}


Other evaluation techniques

[Explanation]

\section{Recommendations}

Synthesis of recommendations

Decision of the sponosoring organisation and its justification

\begin{tabular}{l|l}
\hline 1 & [Explanation] \\
\hline 2 & \\
\hline & \\
\hline
\end{tabular}

and

Overall recommendation

\begin{tabular}{|l|l|l|}
\hline$\square$ & to deploy the initiative without changes \\
\hline$\square$ & to modify the initiative & [Specify how] \\
\hline$\square$ & to cancel the initiative & [Specify why] \\
\hline
\end{tabular}

Decision of the sponosoring organisation and its justification

\section{Comments}

[Explanation] 


\section{Ongoing Steps for Phase II}

\section{Step A: Stakeholder involvement}

\section{Internal stakeholders}

\begin{tabular}{|c|c|c|c|}
\hline Category of stakeholder & $\begin{array}{l}\text { What information } \\
\text { has been } \\
\text { communicated to } \\
\text { stakeholders? }\end{array}$ & $\begin{array}{l}\text { What input have } \\
\text { the stakeholders } \\
\text { provided } \\
\text { (e.g. opinion)? }\end{array}$ & $\begin{array}{l}\text { How was their input } \\
\text { included? Why was } \\
\text { it rejected? }\end{array}$ \\
\hline Data processor(s) & & & \\
\hline Data protection officer(s) (DPO) & & & \\
\hline Recipient(s) (Article 4(9)) & & & \\
\hline Third parties (Article 4(10)) & & & \\
\hline Representative(s) (Article 27) & & & \\
\hline Information security officer(s) & & & \\
\hline Legal service & & & \\
\hline $\begin{array}{l}\text { Employees, trade unions, contrac- } \\
\text { tors, etc. }\end{array}$ & & & \\
\hline [other, specify] & & & \\
\hline
\end{tabular}

\section{External stakeholders}

Category of stakeholder

Data subjects, including:
Minors
Vulnerable persons
[other, specify]

$\begin{array}{lll}\text { What informa- } & \text { What input have } & \text { How was their } \\ \text { tion has been } & \text { the stakeholders } & \text { input included? } \\ \text { communicated to } & \text { provided } & \text { Whywas it } \\ \text { stakeholders? } & \text { (e.g.opinion)? } & \text { rejected? }\end{array}$




\begin{tabular}{|c|c|c|c|c|}
\hline & Category of stakeholder & $\begin{array}{l}\text { What informa- } \\
\text { tion has been } \\
\text { communicated to } \\
\text { stakeholders? }\end{array}$ & $\begin{array}{l}\text { What input have } \\
\text { the stakeholders } \\
\text { provided } \\
\text { (e.g. opinion)? }\end{array}$ & $\begin{array}{l}\text { How was their } \\
\text { input included? } \\
\text { Why was it } \\
\text { rejected? }\end{array}$ \\
\hline \multirow{3}{*}{ 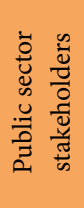 } & Supervisory authority(ies) (DPA) & & & \\
\hline & Policymakers & & & \\
\hline & Local stakeholders & & & \\
\hline \multirow{2}{*}{ 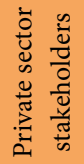 } & Technology providers & & & \\
\hline & Transportation companies & & & \\
\hline \multirow{5}{*}{ 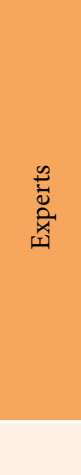 } & $\begin{array}{l}\text { Research Ethics Committees, } \\
\text { within public or private } \\
\text { organisations }\end{array}$ & & & \\
\hline & $\begin{array}{l}\text { National ethics committees or } \\
\text { councils, at EU or Member State } \\
\text { level }\end{array}$ & & & \\
\hline & $\begin{array}{l}\text { Groups of ad hoc recruited ethics } \\
\text { experts }\end{array}$ & & & \\
\hline & Scientific experts & & & \\
\hline & [Anybody else affected, etc., specify] & & & \\
\hline
\end{tabular}

\section{Lack of stakeholder involvement in the present phase}

[If stakeholders are not involved in the present phase of the impact assessment process, explain why.] 
Border Control and New Technologies

Step B: Quality control

Quality control body

What feedback was received?

How was the feedback implemented? Why was it rejected?

Data protection officer(s) (DPO)

Supervisory authority (DPA)

[Other, specify]

\section{Comments}

[Explanation] 


\section{Phase III: Ex post (eventual) steps}

\section{Step 7: Prior Consultation}

\begin{tabular}{|c|c|}
\hline \multirow{6}{*}{ 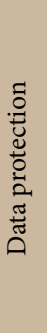 } & Competent DPA(s) \\
\hline & Date of submission \\
\hline & Date of receipt of the response \\
\hline & Inquiry (summary) \\
\hline & Response (summary) \\
\hline & Decision of the controller after consultation \\
\hline \multirow{5}{*}{ 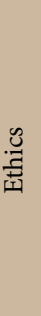 } & Ethics committee and/or competent authority \\
\hline & Date of submission of application for approval \\
\hline & Date of receipt of the response \\
\hline & Response (summary) \\
\hline & $\begin{array}{l}\text { Decision of the sponsoring organisation after } \\
\text { consultation }\end{array}$ \\
\hline
\end{tabular}

\section{Comments}

[Explanation] 


\section{Step 8: Revisiting}

\begin{tabular}{|c|c|c|c|}
\hline \multicolumn{2}{|l|}{ Criterion } & Change? & Explanation \\
\hline \multicolumn{2}{|c|}{$\begin{array}{l}\text { Nature } \\
\text { (what types of processing operations? e.g. collection, storage, } \\
\text { erasure, etc.) }\end{array}$} & $\square$ & \\
\hline \multirow{2}{*}{ Scope } & $\begin{array}{l}\text { Scale } \\
\text { (how much? how many? how far?) }\end{array}$ & $\square$ & \\
\hline & $\begin{array}{l}\text { Time } \\
\text { (when? how long?) }\end{array}$ & $\square$ & \\
\hline \multirow{2}{*}{$\begin{array}{l}\text { Context } \\
\text { (in what circum- } \\
\text { stances?) }\end{array}$} & Internal (concerning the controller) & $\square$ & \\
\hline & $\begin{array}{l}\text { External (concerning individuals, } \\
\text { groups, society, etc.) }\end{array}$ & $\square$ & \\
\hline \multicolumn{2}{|c|}{$\begin{array}{l}\text { Purpose } \\
\text { of processing operations, including, where applicable, } \\
\text { legitimate interest (why?) }\end{array}$} & $\square$ & \\
\hline \multirow{3}{*}{$\begin{array}{l}\text { Benefits } \\
\text { of processing } \\
\text { operations }\end{array}$} & $\begin{array}{l}\text { for individuals, including data } \\
\text { subjects }\end{array}$ & $\square$ & \\
\hline & for the data controller & $\square$ & \\
\hline & for society as a whole & $\square$ & \\
\hline \multirow{3}{*}{$\begin{array}{l}\text { Drawbacks } \\
\text { of processing } \\
\text { operations }\end{array}$} & $\begin{array}{l}\text { for individuals, including data } \\
\text { subjects }\end{array}$ & $\square$ & \\
\hline & for the data controller & $\square$ & \\
\hline & for society as a whole & $\square$ & \\
\hline \multicolumn{2}{|c|}{$\begin{array}{l}\text { Categories of personal data (what?) } \\
\text { special categories of personal data } \\
\text { - personal data of vulnerable persons (e.g. children) } \\
\text { data of a highly personal nature }\end{array}$} & $\square$ & \\
\hline \multicolumn{2}{|c|}{ Means of processing (infrastructure) (by what means?) } & $\square$ & \\
\hline \multicolumn{2}{|c|}{ Envisioned data flows (where to where? whom to whom?) } & $\square$ & \\
\hline \multicolumn{2}{|c|}{ Data security (how is it ensured?) } & $\square$ & \\
\hline \multicolumn{2}{|c|}{ Jurisdiction/market (where?) } & $\square$ & \\
\hline \multicolumn{2}{|c|}{ Actors in the 'supply chain' (who?) } & $\square$ & \\
\hline \multicolumn{2}{|l|}{ [Other, explain] } & $\square$ & \\
\hline
\end{tabular}




\section{Overall suggestion}

What should be done

with the assessment process?

When?

[Specify]

$\square$ revise

$\square$ entirely

$\square$ in part [Specify]

$\square$ do not revise

[Specify why]

\section{[Specify]}

Decision of the sponsoring organisation and its justification 


\section{Ongoing Steps for Phase III}

\section{Step A: Stakeholder involvement}

\section{Internal stakeholders}

\begin{tabular}{|c|c|c|c|}
\hline Category of stakeholder & $\begin{array}{l}\text { What information } \\
\text { has been communi- } \\
\text { cated to stakeholders? }\end{array}$ & $\begin{array}{l}\text { What input have the } \\
\text { stakeholders provi- } \\
\text { ded (e.g. opinion)? }\end{array}$ & $\begin{array}{l}\text { How was their input } \\
\text { included? Why was } \\
\text { it rejected? }\end{array}$ \\
\hline Data processor(s) & & & \\
\hline Data protection officer(s) (DPO) & & & \\
\hline Recipient(s) (Article 4(9)) & & & \\
\hline Third parties (Article 4(10)) & & & \\
\hline Representative(s) (Article 27) & & & \\
\hline Information security officer(s) & & & \\
\hline Legal service & & & \\
\hline $\begin{array}{l}\text { Employees, trade unions, contrac- } \\
\text { tors, etc. }\end{array}$ & & & \\
\hline [other, specify] & & & \\
\hline
\end{tabular}

\section{External stakeholders}

Category of stakeholder

Data subjects, including:
[other, specify]

$\begin{array}{lll}\text { What informa- } & \text { What input have } & \text { How was their } \\ \text { tion has been } & \text { the stakeholders } & \text { input included? } \\ \text { communicated to } & \text { provided (e.g. } & \text { Why was it } \\ \text { stakeholders? } & \text { opinion)? } & \text { rejected? }\end{array}$

opinion)? 


\begin{tabular}{|l|l} 
& Category of stakeholder \\
\hline & Supervisory authority(ies) (DPA) \\
\hline & Policymakers \\
\hline & Technology providers \\
\hline & Transportation companies \\
\hline & $\begin{array}{l}\text { Research Ethics Committees, } \\
\text { within public or private orga- }\end{array}$ \\
\hline nisations
\end{tabular}

\section{Lack of stakeholder involvement in the present phase}

[If stakeholders are not involved in the present phase of the impact assessment process, explain why.] 
Border Control and New Technologies

\section{Step B: Quality control}

Quality control body

What feedback was received?

How was the feedback implemented?

Why was it rejected?

Data protection officer(s) (DPO)

Supervisory authority (DPA)

[Other, specify]

\section{Comments}

[Explanation] 


\section{Step C: Documentation}

\begin{tabular}{|c|c|c|c|c|c|}
\hline & & Attachment & $\begin{array}{c}\text { Confidentiality } \\
\text { level }\end{array}$ & 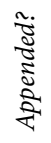 & Comments \\
\hline \multirow{2}{*}{$\begin{array}{l}\text { Step } 1 \\
\text { Step } 4\end{array}$} & \multirow{10}{*}{ 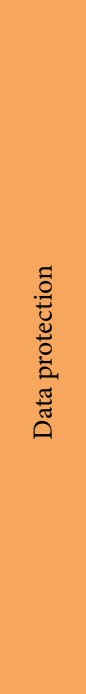 } & \multirow{2}{*}{ Record of processing activities } & & $\square$ & \\
\hline & & & & $\square$ & \\
\hline \multirow{7}{*}{ Step 2} & & Approved codes of conduct & & $\square$ & \\
\hline & & Certificates & & $\square$ & \\
\hline & & Binding corporate rules (BCRs) & & $\square$ & \\
\hline & & $\begin{array}{l}\text { Standard contractual clauses } \\
\text { (SCCs) }\end{array}$ & & $\square$ & \\
\hline & & Data protection policies & & $\square$ & \\
\hline & & Professional codes of conduct & & $\square$ & \\
\hline & & Data sharing agreement(s) & confidential & $\square$ & \\
\hline \multirow{3}{*}{ Step 3} & & $\begin{array}{l}\text { A copy of a service contract } \\
\text { (in the event that the impact } \\
\text { assessment is outsourced) }\end{array}$ & & $\square$ & \\
\hline & \multirow{2}{*}{ 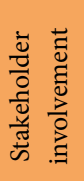 } & $\begin{array}{l}\text { A list of stakeholders to consult } \\
\text { and their contact details }\end{array}$ & & $\square$ & \\
\hline & & Stakeholder consultation plan & confidential & $\square$ & \\
\hline \multirow{3}{*}{ Step 7} & \multirow{3}{*}{ 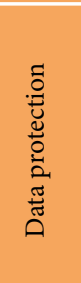 } & $\begin{array}{l}\text { Request for prior consultation } \\
\text { with a supervisory authority }\end{array}$ & & $\square$ & \\
\hline & & $\begin{array}{l}\text { Response from a supervisory } \\
\text { authority }\end{array}$ & & $\square$ & \\
\hline & & $\begin{array}{l}\text { Response from a supervisory } \\
\text { authority }\end{array}$ & & $\square$ & \\
\hline
\end{tabular}




\begin{tabular}{|c|c|c|c|c|c|}
\hline & & Attachment & $\begin{array}{c}\text { Confidentiality } \\
\text { level }\end{array}$ & 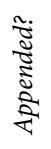 & Comments \\
\hline \multirow{3}{*}{ Step A } & \multirow{2}{*}{ 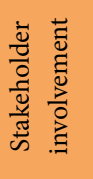 } & $\begin{array}{l}\text { Technical briefing(s) for stake- } \\
\text { holder consultation }\end{array}$ & & $\square$ & \\
\hline & & $\begin{array}{l}\text { Stakeholder consultation } \\
\text { (reports) }\end{array}$ & & $\square$ & \\
\hline & 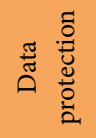 & DPO opinion (report) & & $\square$ & \\
\hline \multicolumn{3}{|c|}{ [Reports from other evaluation techniques; specify] } & & $\square$ & \\
\hline \multicolumn{3}{|c|}{ [other, explain] } & & $\square$ & \\
\hline
\end{tabular}

\section{Comments}

[Explanation] 
Closing Page

\section{Endorsements}

\begin{tabular}{|c|c|c|c|c|}
\hline Responsibility & Name & Remarks & Date & Signature \\
\hline \multicolumn{5}{|l|}{ Assessor(s) } \\
\hline \multicolumn{5}{|c|}{ Data protection officer } \\
\hline \multicolumn{5}{|c|}{ Data controller(s) } \\
\hline [other, explain] & & & & \\
\hline
\end{tabular}

\section{Endnotes}

1. Based on: Dariusz Kloza et al., "Data Protection Impact Assessment in the European Union: Developing a Template for a Report from the Assessment Process," d.pia.lab Policy Brief (Brussels: VUB, 2020), https://doi.org/10.31228/osf.io/7qrfp. 